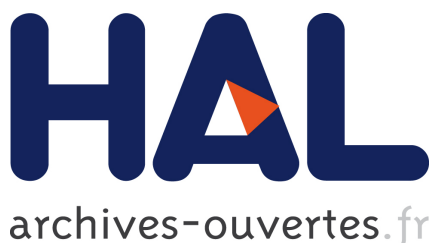

\title{
Optimized diffusion approximation
}

\author{
Ugo Tricoli, Callum Macdonald, Anabela da Silva, Vadim A. Markel
}

\section{To cite this version:}

Ugo Tricoli, Callum Macdonald, Anabela da Silva, Vadim A. Markel. Optimized diffusion approximation. Journal of the Optical Society of America. A Optics, Image Science, and Vision, Optical Society of America, 2018, 35 (2), pp.356. 10.1364/JOSAA.35.000356 . hal-02376510

\section{HAL Id: hal-02376510 https://hal.archives-ouvertes.fr/hal-02376510}

Submitted on 26 Nov 2019

HAL is a multi-disciplinary open access archive for the deposit and dissemination of scientific research documents, whether they are published or not. The documents may come from teaching and research institutions in France or abroad, or from public or private research centers.
L'archive ouverte pluridisciplinaire HAL, est destinée au dépôt et à la diffusion de documents scientifiques de niveau recherche, publiés ou non, émanant des établissements d'enseignement et de recherche français ou étrangers, des laboratoires publics ou privés. 


\title{
Optimized diffusion approximation
}

\author{
Ugo Tricoli, Callum M. Macdonald , Anabela Da Silva, and Vadim A. Markel* \\ ${ }^{1}$ Aix Marseille Univ, CNRS, Centrale Marseille, Institut Fresnel, F-13013 Marseille, France \\ *Corresponding author: vmarkel@pennmedicine.upenn.edu
}

Compiled December 20, 2017

\begin{abstract}
We show that the diffusion approximation to the radiative transport equation, which is commonly used in biomedical optics to describe propagation of light in tissues, contains a previously unexplored adjustable parameter. This parameter is related to the rate of exponential decay of the reduced intensity. In the conventional theories, there are two distinct choices for this parameter. However, neither of these choices are optimal. When the optimal value for the parameter is used, the resulting DA becomes much more accurate near the medium boundaries, e.g., at the depth of up to a few $\ell^{*}$, where $\ell^{*}$ is the transport mean free path (typically, about $1 \mathrm{~mm}$ in tissues). We refer to the new adjustable parameter as to the reduced extinction coefficient. The proposed technique can reduce the relative error of the predicted diffuse density of the optical energy from about $30 \%$ to less than $1 \%$. The optimized diffusion approximation can still be inaccurate very close to an interface or in some other physical situations. Still the proposed development extends the applicability range of the diffusion approximation significantly. This result can be useful, for instance, in tomographic imaging of relatively shallow (up to a few $\ell^{*}$ deep) layers of tissues in the reflection geometry.
\end{abstract}

OCIS codes: (010.5620) Radiative transfer; (290.1990) Diffusion; (170.3660) Light propagation in tissues

http://dx.doi.org/10.1364/JOSAA.XX.XXXXXX

\section{INTRODUCTION}

Derivation of the diffusion approximation (DA) to the Boltzmann's equation is a fundamental theoretical result, which explains the emergence of diffusion phenomena from complex non-equilibrium kinetics. In the context of biomedical optics, the diffusion equation (DE) is frequently used to approximate the solutions to the stationary or time-dependent radiative transport equation (RTE). Many optical imaging modalities such as diffuse optical tomography $[1,2]$ are based on inverting the DE. Even though inversion of the RTE has also been developed [3-5], DA remains the mainstay of optical tomography.

While various derivations of the DA have been known for a long time (e.g., see [6]), a detailed investigation of the subject started in the 1990s motivated by the newly emerging application in optical tomography and imaging. It has been generally understood that the DA is applicable when $\mu_{a} \ll \mu_{s}$, where $\mu_{a}$ and $\mu_{s}$ are the absorption and scattering coefficients of the medium [? ], and then only sufficiently far from the source of radiation (assumed to be localized) and from the medium boundaries. However, the exact conditions of applicability of the DA or the associated error estimates proved to be difficult to obtain.

In part, this is due to a number of uncertainties inherent in the various derivations of the DA. One such uncertainty is related to the definition of the diffusion coefficient $D$ [7-10]. There exist different methods of defining $D$ : by using the so-called $P_{1}$ approximation [11], or by asymptotic analysis [12], or by computing the largest "diffuse" eigenvalue in the discrete spectrum of the linear operator of the RTE [13]. All these methods yield somewhat different results and the discrepancy can be significant if the ratio $\mu_{a} / \mu_{s}$ is not small. Some comparison of different definitions can be found in $[7,14]$. However, in the case of biological tissues in the near-IR spectral range, $\mu_{a} / \mu_{S} \sim 10^{-5}$, and the above problem is not significant. In this paper, we use the definition $D=\ell^{*} / 3$, where $\ell^{*}$ is the photon transport mean free path (defined below); other definitions will not be considered.

Another uncertainty is related to the boundary conditions for the DE at diffuse-nondiffuse interfaces. The most general mixed boundary condition admitted by the DE contains the so-called extrapolation distance parameter $\ell$. One naturally wishes to find the value of $\ell$ that results in the best fit between the DE and RTE solutions. Analytical but somewhat ad hoc expressions for the optimal $\ell$ have been obtained [11,15-17] (some of them are discussed below), but it seems equally reasonable to treat $\ell$ as an adjustable parameter. A more accurate description of propagation of multiply-scattered light near such interfaces is based on the theory of boundary layers [12]. However, this theory involves functions that are not obtained from a DE. Recently, a new method for treating the boundary layers was proposed [18] in which the diffusion coefficient is allowed to change close to 
the interface even though the medium is physically uniform. While this can yield accurate results for reflection or transmission, it is not clear how this approach can be used in the context of tomography.

Finally, one needs to determine the correct source term for the DE. This problem is nontrivial because the source term for the RTE is a function of the form $\varepsilon(\mathbf{r}, \hat{\mathbf{s}})$, where $\mathbf{r}$ is the radius vector of position and $\hat{s}$ is a unit vector of direction. In contrast, the source term for the DE is of the form $S(\mathbf{r})$ and, therefore, it has less degrees of freedom than $\varepsilon(\mathbf{r}, \hat{\mathbf{s}})$. This fundamental mathematical difficulty is addressed in the diffusion theory by defining source functions that mimic the $\hat{\mathbf{s}}$-dependence of $\varepsilon(\mathbf{r}, \hat{\mathbf{s}})$ by the shape of $S(\mathbf{r})$ [18-21].

In this paper, we focus on the problem of correct determination of $S(\mathbf{r})$. This problem is closely related to decomposition of the total specific intensity (from now on, simply, intensity) into the reduced and diffuse components. There are infinitely many ways in which this can be done and, in each case, a different expression for the source term $S(\mathbf{r})$ is obtained. The two conventional definitions of the reduced intensity are discussed in Sec. 2. More generally, it can be shown that the decomposition in question is governed by an additional adjustable parameter, which is denoted below by $\bar{\mu}$ [13]. We will refer to $\bar{\mu}$ as to the reduced extinction coefficient. This parameter remains largely unexplored in the literature. In this paper, we treat $\bar{\mu}$ (and also the extrapolated boundary distance $\ell$ ) as adjustable parameters. We then optimize these two parameters using rigorous RTE solutions as a benchmark and show that the precision of the resulting DA near diffuse-nondiffuse interfaces can be noticeably improved compared to the standard theory. We emphasize that the main contribution to this improvement comes from optimizing $\bar{\mu}$ as we obtain the conventional results for the optimal $\ell$. However, the optimal value of $\bar{\mu}$ is not universal; it depends on the details of the phase function even if the scattering asymmetry parameter $g$ is fixed. Further, the optimization performed in this paper is applicable to the normal and close to normal incidences only. An optimized DA equally applicable to all angles of incidence likely does not exist.

Our approach can be viewed as an approximate theory of transition layers because the reduced intensity decays exponentially with depth. The results of this paper can be important for optical imaging modalities involving diffuse reflection measurements, especially when relatively shallow (up to a few transport mean free paths) concentration of a fluorescent contrast agent is the quantity of interest. In this case, the rate of excitation of the fluorophores is proportional to the local density of electromagnetic energy $u(\mathbf{r})$ [22], and we will seek the reduced extinction coefficient $\bar{\mu}$ that yields the most accurate approximation for $u(\mathbf{r})$.

The paper is organized as follows. Derivation of the optimized DA is given in section 2 and its relation to the traditional theories are discussed in sections 3 . Sections 4, 5, 6, contain numerical examples for one-dimensional propagation (incident plane wave illumination). In section 7 three-dimensional solutions are considered for a incident pencil beam of small but finite radius. All numerical solutions to the RTE that are used as benchmarks have been obtained by Monte Carlo simulations (we assumed no index mismatch at the diffuse-nondiffuse interface). Finally, section 8 contains a discussion of the obtained results.

\section{OPTIMIZED DIFFUSION APPROXIMATION}

The mathematical point of departure for developing the optimized DA is the RTE, which we write here in the stationary form as

$$
\left(\hat{\mathbf{s}} \cdot \nabla+\mu_{t}\right) I(\mathbf{r}, \hat{\mathbf{s}})=\mu_{s} \int A\left(\hat{\mathbf{s}}, \hat{\mathbf{s}}^{\prime}\right) I\left(\mathbf{r}, \hat{\mathbf{s}}^{\prime}\right) d^{2} s^{\prime}+\varepsilon(\mathbf{r}, \hat{\mathbf{s}}) .
$$

Here $\mu_{t}=\mu_{s}+\mu_{a}$ is the extinction coefficient, $\mu_{s}$ and $\mu_{a}$ are the scattering and absorption coefficients, $A\left(\hat{\mathbf{s}}, \hat{\mathbf{s}}^{\prime}\right)$ is the phase function and $\varepsilon(\mathbf{r}, \hat{\mathbf{s}})$ is the RTE source. The medium is assumed to be isotropic on average, which implies that $A\left(\hat{\mathbf{s}}, \hat{\mathbf{s}}^{\prime}\right)=f\left(\hat{\mathbf{s}} \cdot \hat{\mathbf{s}}^{\prime}\right)$, and the phase function is normalized by the condition

$$
\int A\left(\hat{\mathbf{s}}, \hat{\mathbf{s}}^{\prime}\right) d^{2} s^{\prime}=2 \pi \int_{-1}^{1} f(x) d x=1 .
$$

In this paper, $d^{2} s$ denotes the element of solid angle about the direction of the unit vector $\hat{\mathbf{s}}$, that is, $d^{2} s=\sin \theta d \theta d \varphi$, where $\theta$ and $\varphi$ are the polar and azimuthal angles of $\hat{\mathbf{s}}$ in the laboratory frame.

Similarly to the conventional theory, the optimized DA involves a decomposition of the total intensity into the reduced and diffuse components, $I=I_{r}+I_{d}$, where the reduced component is ballistic and propagates through the medium without scattering. The physical idea behind this decomposition is that the total intensity, especially close to the source, always has a highly singular component that can not be described by a DA. This singular component is included in $I_{r}$. In contrast, the diffuse component is assumed to be a smooth function of $\hat{\mathbf{s}}$.

As was noted in [13], the splitting of the total intensity into the diffuse and reduced parts is rather arbitrary. In general, the reduced intensity is defined by the equation

$$
(\hat{\mathbf{s}} \cdot \nabla+\bar{\mu}) I_{r}(\mathbf{r}, \hat{\mathbf{s}})=\varepsilon(\mathbf{r}, \hat{\mathbf{s}}),
$$

where $\bar{\mu}$ is the rate of exponential decay of $I_{r}$ away from the source (the reduced extinction coefficient). The value of $\bar{\mu}$ is so far undetermined and we argue that it can not be determined theoretically, at least not without considering the complicated theory of boundary layers in transport problems, which we wish to avoid here.

Still, to build a usable approximation, one needs to make some choice for $\bar{\mu}$. The most common such choice $\bar{\mu}=\mu_{t}=\mu_{a}+$ $\mu_{s}$ is due to Ishimaru [23]. To justify this choice, one can imagine that $I_{r}$ gives the fraction of incident radiation ("photons") that are neither absorbed nor scattered up to a given propagation distance. In this sense, the above definition of $\bar{\mu}$ is consistent with the common understanding of the term "extinction". For the same reason, $I_{r}$ is sometimes referred to as the coherent component of the intensity, even though this terminology is not precise.

However, the physical intuition fails us in this instance and the choice $\bar{\mu}=\mu_{t}$ (that is, the reduced and the total extinction coefficients are the same) is in fact problematic. Indeed, $\mu_{t}$ does not characterize the absorption and scattering in the medium completely; there is also the phase function. If the phase function is highly forward-peaked, $\mu_{t}$ ceases to be a meaningful parameter. Indeed, consider the case of biological tissues in the near-IR spectral range, for which $\mu_{a} / \mu_{t} \sim 6 \cdot 10^{-5}$ but single scattering occurs predominantly in a narrow cone whose axis is the incident direction. It is clear that many photons will change their direction upon single scattering only slightly and will not be noticeably different from the photons that did not experience scattering at all. In this case, a more reasonable choice for $\bar{\mu}$ 
seems to be in between $\mu_{a}$ and $\mu_{t}$, which differ by several orders of magnitude.

We note that, in Ishimaru's classical exposition of the subject [23], there is an additional subtle point, which is not typically discussed. Namely, Ishimaru considers the surface and the volume sources for the RTE separately and assumes that only the surface term gives rise to the reduced intensity [? ]. Further, the surface term (which is due to the radiation entering the medium from outside) is not written explicitly but is accounted for through the boundary conditions applied to $I_{r}$. We however do not see a justification for treating the surface and volume sources in the RTE differently. In the optimized DA, both types of sources produce the reduced intensity. Correspondingly, we work below with just one source function $\varepsilon(\mathbf{r}, \hat{\mathbf{s}})$ in Eq. (1), which can include both surface and volume contributions. In this formulation of the problem, the total intensity satisfies homogeneous half-range boundary conditions at all diffuse-nondiffuse interfaces.

The second obvious choice for $\bar{\mu}$ is $\bar{\mu}=\mu^{*}$ where $\mu^{*}=\mu_{a}+$ $(1-g) \mu_{s}$ is reciprocal of the transport mean free path $\ell^{*}$ [24]. Here $g=\int \mathbf{s} \cdot \hat{\mathbf{s}}^{\prime} A\left(\mathbf{s}, \hat{\mathbf{s}}^{\prime}\right) d^{2} s^{\prime}=2 \pi \int_{-1}^{1} x f(x) d x$ is the scattering asymmetry parameter. This choice is free from the paradox mentioned above: in the limit $g \rightarrow 1$ (highly forward-peaked scattering), $\bar{\mu}$ approaches $\mu_{a}$ rather than $\mu_{t}$, as one can expect on physical grounds. In addition, this choice results in the source function for the DE of a particularly simple form (see below).

What is important for us here is that neither of the two choices for the reduced extinction coefficient $\bar{\mu}$ described above have been rigorously justified. We therefore find it more logical to treat $\bar{\mu}$ as an adjustable parameter (together with the extrapolation distance $\ell$, defined precisely below). We therefore are not making any assumptions about $\bar{\mu}$ at this point and proceed with the derivation.

As in all conventional diffusion theories, we seek diffuse component of the intensity in the form [?]

$$
I_{d}(\mathbf{r}, \hat{\mathbf{s}}) \approx \frac{1}{4 \pi}\left[u_{d}(\mathbf{r})+3 \hat{\mathbf{s}} \cdot \mathbf{J}_{d}(\mathbf{r})\right],
$$

where

$$
u_{d}(\mathbf{r})=\int I_{d}(\mathbf{r}, \hat{\mathbf{s}}) d^{2} s, \mathbf{J}_{d}(\mathbf{r})=\int \hat{\mathbf{s}} I_{d}(\mathbf{r}, \hat{\mathbf{s}}) d^{2} s .
$$

are the diffuse density and current of energy. We then substitute the decomposition $I=I_{r}+I_{d}$, where $I_{d}$ is given by Eq. (4) and $I_{r}$ satisfies Eq. (3), into Eq. (1). Since we have used an ansatz for $I_{d}$ that is not of sufficiently general form, the resulting equation will not generally hold. However, one can require that the zeroth and first angular moments (integrals with respect to $d^{2} s$ and $\hat{\mathbf{s}} d^{2} s$ ) of this equation hold. From this requirement, we obtain the following set of differential equations for $u_{d}$ and $\mathbf{J}_{d}$ :

$$
\begin{aligned}
& \nabla \cdot \mathbf{J}_{d}+\mu_{a} u_{d}=E \equiv\left(\bar{\mu}-\mu_{a}\right) u_{r}, \\
& \frac{1}{3} \nabla u_{d}+\mu^{*} \mathbf{J}_{d}=\mathbf{Q} \equiv\left(\bar{\mu}-\mu^{*}\right) \mathbf{J}_{r},
\end{aligned}
$$

where

$$
u_{r}(\mathbf{r})=\int I_{r}(\mathbf{r}, \hat{\mathbf{s}}) d^{2} s, \quad \mathbf{J}_{r}(\mathbf{r})=\int \hat{\mathbf{s}} I_{r}(\mathbf{r}, \hat{\mathbf{s}}) d^{2} s
$$

are the reduced density and current of energy. The latter quantities can usually be found analytically by solving Eq. (3) and substituting the solution into Eq. (7). Explicit forms of the scalar and vector source functions $E$ and $\mathbf{Q}$ are given below for some special cases. The total density and current are given by the sums of the reduced and diffuse components, that is, $u=u_{r}+u_{d}$ and $\mathbf{J}=\mathbf{J}_{r}+\mathbf{J}_{d}$.

We can eliminate $\mathbf{J}_{d}$ from Eq. (6) to obtain a second-order equation containing $u_{d}$ only:

$$
-\nabla \cdot D \nabla u_{d}+\mu_{a} u_{d}=S,
$$

where $D=\ell^{*} / 3$ is the diffusion coefficient and

$$
S=E-\ell^{*} \nabla \cdot \mathbf{Q}
$$

is the source for the DE. The presence of the derivative in Eq. (9) can lead to some confusion. Indeed, the differentiation can result in appearance of a delta-function, and this delta-function can be centered exactly at the medium boundary. In this case, it is not immediately obvious whether this delta-function should be included in the source function or not. While this question can be addressed, we find it easier to work with the first-order Eq. (6).

The latter, however, must be complemented by a boundary condition. The function given in Eq. (4) can not satisfy the halfrange boundary condition of the RTE. Therefore, this rigorous condition is customarily replaced by the equation [23]

$$
\left.\int_{\hat{\mathbf{n}} \cdot \hat{\mathbf{s}} \leq 0}(\hat{\mathbf{n}} \cdot \hat{\mathbf{s}}) I_{d}(\mathbf{r}, \hat{\mathbf{s}}) d^{2} S\right|_{\mathbf{r} \in \partial \Omega}=0,
$$

where $\hat{\mathbf{n}}$ is the outward unit normal to the boundary $\partial \Omega$ of the region $\Omega$ occupied by the medium. Substituting the decomposition Eq. (4) into Eq. (10), we obtain

$$
\left.\left(u_{d}-2 \hat{\mathbf{n}} \cdot \mathbf{J}_{d}\right)\right|_{\mathbf{r} \in \partial \Omega}=0 .
$$

This is a special case of the more general boundary condition

$$
\left.\left(u_{d}-3 \frac{\ell}{\ell^{*}} \hat{\mathbf{n}} \cdot \mathbf{J}_{d}\right)\right|_{\mathbf{r} \in \partial \Omega}=0,
$$

where $\ell$ is an adjustable parameter and the factor $3 / \ell^{*}$ has been introduced for convenience. The boundary condition given in Eq. (11) is recovered if we take $\ell=2 \ell^{*} / 3$, which is one the conventional values for $\ell$ if there is no refractive index mismatch and no Fresnel reflections at the boundary (another commonlyencountered choice is $\left.\ell=0.71 \ell^{*}\right)$.

Equations (6) and (12) form the mathematical basis of the optimized DA.

\section{DIFFUSION APPROXIMATION WITHOUT THE VEC- TOR SOURCE}

While Eqs. (6),(12) have been derived above in a straightforward manner, they might look not very familiar due to the presence of the vector source term $\mathbf{Q}$. Note that the boundary condition equivalent to Eq. (12) but involving $u_{d}$ only is of the form

$$
\left.\left(u_{d}+\ell \hat{\mathbf{n}} \cdot \nabla u_{d}-3 \ell \hat{\mathbf{n}} \cdot \mathbf{Q}\right)\right|_{\mathbf{r} \in \partial \Omega}=0,
$$

which is inhomogeneous and different from the frequentlyencountered homogeneous boundary condition

$$
\left.\left(u_{d}+\ell \hat{\mathbf{n}} \cdot \nabla u_{d}\right)\right|_{\mathbf{r} \in \partial \Omega}=0 .
$$

While it is known that the correct boundary condition in a DA contain a free term [7, 12], the homogeneous condition (14) is used far more frequently in the optical tomography literature. In fact, the questions of choosing the right boundary condition 
and the source term for the DA are closely related and should not be considered separately.

One of the goals of this paper is to provide a clear and mathematically-consistent derivation of the source term and the corresponding boundary condition. To this end, it appears instructive to consider an alternative formulation that does not contain the vector source $\mathbf{Q}$. Of course, $\mathbf{Q}=0$ if $\bar{\mu}=\mu^{*}$. But we will consider now a more general approach to removing $\mathbf{Q}$ from consideration, which does not use the assumption $\bar{\mu}=\mu^{*}$.

The basic idea behind this approach can be loosely summarized as follows. First, we can shift the support of the source functions $E(\mathbf{r})$ and $\mathbf{Q}(\mathbf{r})$ into the medium by an infinitesimal distance so that it no longer touches the boundary, and this operation should not influence the solution to the DE substantially, certainly, not sufficiently deep inside the medium. Then $\mathbf{Q}(\mathbf{r})=0$ if $\mathbf{r} \in \partial \Omega$ [? ]. Therefore, the shift results in Eq. (8) with $S$ given by (9) and the homogeneous boundary condition (14). However, the source term $S$ in Eq. (8) still depends on $\mathbf{Q}$.

The next step is to note that the function $S(\mathbf{r})$ might be quite complicated and not known precisely but, apparently, all that matters are the moments $M_{0}=\int S(\mathbf{r}) d^{3} r$ and $\mathbf{M}_{1}=$ $\int\left(\mathbf{r}-\mathbf{r}_{s}\right) S(\mathbf{r}) d^{3} r$, where $\mathbf{r}_{S} \in \partial \Omega$ is the point at which an infinitely narrow incident beam enters the medium (the source location). For the one-dimensional propagation problem, which is considered in sections 4 through 6 of this paper, the moments are defined as $M_{0}=\int S(z) d z$ and $\mathbf{M}_{1}=\hat{\mathbf{z}} \int z S(z) d z$, where $z$ is the depth direction and the radiation enters the medium through the plane $z=0$. For a more general three-dimensional case, we can write [25]

$$
\begin{aligned}
u(\mathbf{r}) & =\int G\left(\mathbf{r}, \mathbf{r}^{\prime}\right) S\left(\mathbf{r}^{\prime}\right) d^{3} r^{\prime} \\
& \approx G\left(\mathbf{r}, \mathbf{r}_{S}\right) M_{0}+\left.\frac{\partial G\left(\mathbf{r}, \mathbf{r}^{\prime}\right)}{\partial \mathbf{r}^{\prime}}\right|_{\mathbf{r}^{\prime}=\mathbf{r}_{S}} \cdot \mathbf{M}_{1},
\end{aligned}
$$

where $G\left(\mathbf{r}, \mathbf{r}^{\prime}\right)$ is the Green's function for Eq. (8), that is, the solution to Eq. (8) for $S(\mathbf{r})=\delta\left(\mathbf{r}-\mathbf{r}^{\prime}\right)$ subject to the homogeneous boundary condition (14) with respect to both arguments. It then follows from Eq. (14) that

$$
\left.\hat{\mathbf{n}} \cdot \frac{\partial G\left(\mathbf{r}, \mathbf{r}^{\prime}\right)}{\partial \mathbf{r}^{\prime}}\right|_{\mathbf{r}^{\prime}=\mathbf{r}_{s}}=-\frac{1}{\ell} G\left(\mathbf{r}, \mathbf{r}_{S}\right) .
$$

In addition, for a normally incident beam,

$$
\mathbf{M}_{1}=-\hat{\mathbf{n}} \frac{\bar{\mu} / \mu^{*}-\mu_{a} / \bar{\mu}}{\bar{\mu}-\mu_{a}} M_{0} .
$$

This relation can be verified by a direct computation keeping in mind that the expression $\nabla \cdot \mathbf{Q}$ contains a delta-function due to the displacement of the support of $\mathbf{Q}$ into the medium, which ensues differentiation of a discontinuous function. We can now rewrite Eq. (15) as

$$
u(\mathbf{r}) \approx\left(1+\frac{1}{\ell} \frac{\bar{\mu} / \mu^{*}-\mu_{a} / \bar{\mu}}{\bar{\mu}-\mu_{a}}\right) G\left(\mathbf{r}, \mathbf{r}_{s}\right) M_{0} .
$$

In other words, the density deep inside the medium is given, up to a constant overall factor, by the Green's function $G\left(\mathbf{r}, \mathbf{r}_{s}\right)$, which is independent of the form of the source. Note that, in the case $\bar{\mu}=\mu^{*}$, the factor in the brackets in the right-hand side of Eq. (18) becomes equal to $1+\ell^{*} / \ell$ in agreement with [25].

So far, we have not really departed from the mathematical formalism of the optimized DA. All we did was to move the support of the functions $E(\mathbf{r})$ and $\mathbf{Q}(\mathbf{r})$ away from the boundary by an infinitesimal distance, so that the boundary condition now does not contain $\mathbf{Q}$ and is of the homogeneous form given by Eq. (14). In particular, Eq. (18) is a valid asymptote in the optimized DA. However, the form of this asymptote suggests that the only trace of $\bar{\mu}$ is in the overall coefficient. This further suggests that we can simplify the formalism by forgetting about the vector source $\mathbf{Q}$ and assuming (without any further justification) that it is zero.

If we follow the above prescription, we will have to solve Eq. (8) in which $S=E$ with the homogeneous boundary condition Eq. (14). We will refer to this approach and the corresponding solutions as to conventional. Of course, the conventional and the optimized approaches coincide exactly if $\bar{\mu}=\mu^{*}$ but, otherwise, they can differ strongly near the boundaries. In the conventional approach, the relation between $M_{0}$ and $\mathbf{M}_{1}$ is of the form

$$
\bar{\mu} \mathbf{M}_{1}=-\hat{\mathbf{n}} M_{0},
$$

which corresponds to the asymptotic solution

$$
u(\mathbf{r}) \approx\left(1+\frac{1}{\bar{\mu} \ell}\right) G\left(\mathbf{r}, \mathbf{r}_{S}\right) M_{0} .
$$

This is of the same functional form as Eq. (18), up to an overall constant. However, we emphasize again that the solutions in the optimized and the conventional DAs are substantially different near the boundaries, and this difference is not reduced to multiplication by a constant.

As mentioned above, we will optimize solutions to Eq. (6) treating $\bar{\mu}$ and $\ell$ as adjustable parameters. For comparison purposes, we will also optimize (with respect to the same parameters) the conventional solutions described above. We reiterate that the conventional solutions are obtained by solving Eq. (8) with $S=E$ (that is, we set $\mathbf{Q}=0$ ) subject to the homogeneous boundary condition Eq. (14).

Finally, note that all the arguments of this section can be repeated for one-dimensional propagation in which all quantities of interest depend only on the depth $z$.

\section{NUMERICAL EXAMPLES FOR ONE-DIMENSIONAL PROPAGATION AND NORMAL INCIDENCE}

Let the scattering medium occupy the half-space $z>0$ and consider a plane wave, normally-incident family of rays. This excitation is described by the RTE source term $\varepsilon(\mathbf{r}, \hat{\mathbf{s}})=W \delta(z) \delta_{2}(\hat{\mathbf{s}}, \hat{\mathbf{z}})$ where $\delta_{2}$ is the angular delta-function and $W$ is the incident energy per unit time per unit area. Then the reduced intensity is of the form $I_{r}(\mathbf{r}, \hat{\mathbf{s}})=W \exp (-\bar{\mu} z) \delta_{2}(\hat{\mathbf{s}}, \hat{\mathbf{z}})$. From this we can compute the reduced density and current of energy according to Eq. (7) and the source terms according to Eq. (6) (second equalities). The result is

$$
\begin{aligned}
E(z) & =W\left(\bar{\mu}-\mu_{a}\right) \exp (-\bar{\mu} z), \\
\mathbf{Q}(z) & =W\left(\bar{\mu}-\mu^{*}\right) \exp (-\bar{\mu} z) \hat{\mathbf{z}} .
\end{aligned}
$$

It can be seen that Eq. (6) takes in this case the form

$$
\begin{aligned}
\frac{\partial J_{d}(z)}{\partial z}+\mu_{a} u_{d}(z) & =W\left(\bar{\mu}-\mu_{a}\right) \exp (-\bar{\mu} z), \\
\frac{1}{3} \frac{\partial u_{d}(z)}{\partial z}+\mu^{*} J_{d}(z) & =W\left(\bar{\mu}-\mu^{*}\right) \exp (-\bar{\mu} z) .
\end{aligned}
$$

Here $J_{d}$ is the Cartesian component of the diffuse current in the $Z$ direction; the other two Cartesian components of the diffuse and 

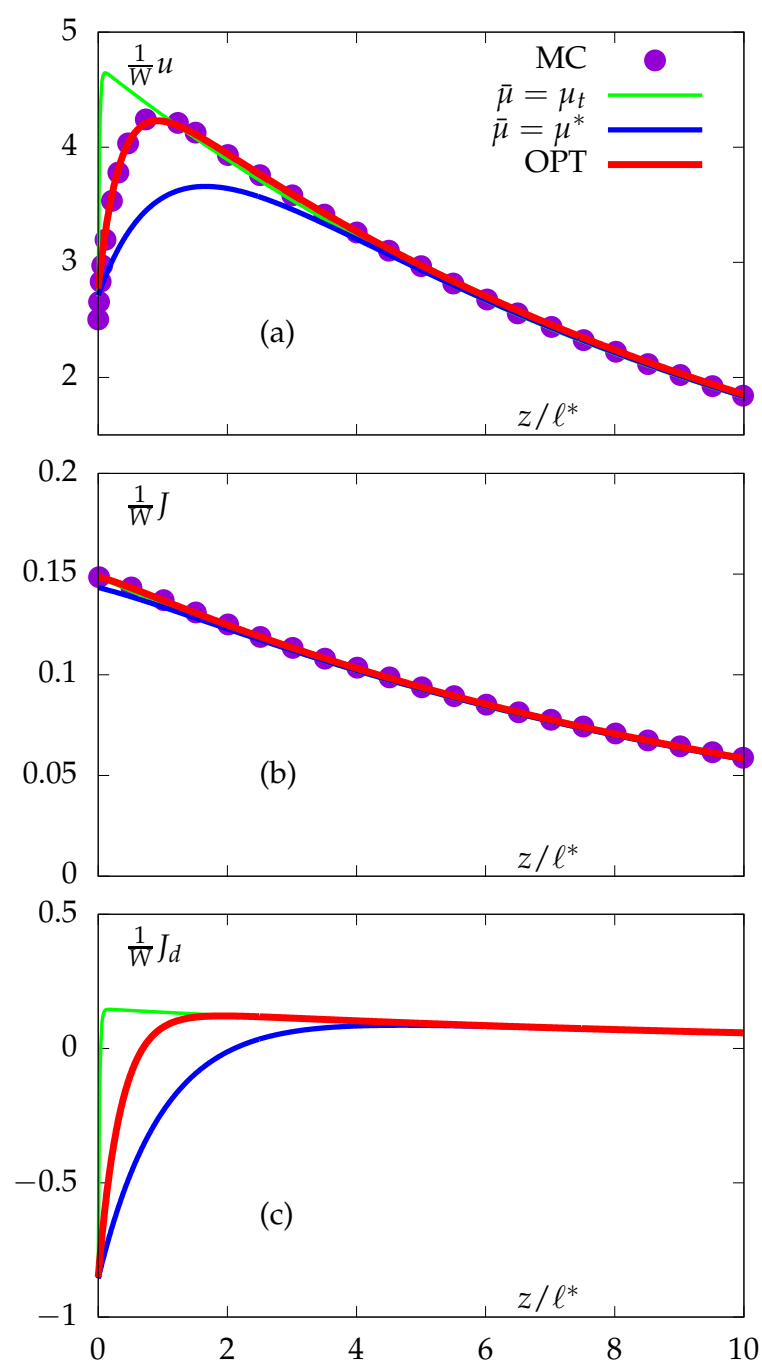

Fig. 1. Total density $u$ (a), total current $J(\mathrm{~b})$ and the diffuse component of the current $J_{d}$ (c) as functions of the normalized depth $z / \ell^{*}$ for one-dimensional propagation in the half space $z>0$. Parameters of the medium are $\mu_{a} / \mu_{s}=0.03 / 500$ and $g=0.98$. The curves $\bar{\mu}=\mu_{t}$ and $\bar{\mu}=\mu^{*}$ were computed according to Eq. (26) with $\bar{\mu}$ as labeled and $\ell=2 \ell^{*} / 3$. The curves labeled "OPT" were computed according to the same formulas but for the optimal values of $\bar{\mu}$ and $\ell$, which are in this case $\bar{\mu}_{\mathrm{opt}}=2.84 \mu^{*}, \ell_{\mathrm{opt}}=0.69 \ell^{*}$.

total currents are zero by symmetry. The boundary condition Eq. (12) takes the form

$$
u_{d}(0)+3 \frac{\ell}{\ell^{*}} J_{d}(0)=0
$$

The solution to Eqs. (22),(23) is

$$
\begin{aligned}
& \frac{1}{W} u_{d}(z)=(A-1) e^{-\bar{\mu} z}+B e^{-k_{d} z} \\
& \frac{1}{W} J_{d}(z)=\left(\frac{\mu_{a}}{\bar{\mu}} A-1\right) e^{-\bar{\mu} z}+\frac{\mu_{a}}{k_{d}} B e^{-k_{d} z},
\end{aligned}
$$

where

$$
\begin{aligned}
& A=-\frac{2 \bar{\mu}^{2}}{\bar{\mu}^{2}-k_{d}^{2}}, k_{d}=\sqrt{3 \mu_{a} \mu^{*}}, \\
& B=\frac{3 \bar{\mu}^{2}-k_{d}^{2}}{\bar{\mu}^{2}-k_{d}^{2}}+\frac{3 \ell}{1+k_{d} \ell}\left[\mu^{*}-\frac{\bar{\mu} k_{d}+\frac{k_{d}^{2}}{3}}{\bar{\mu}+k_{d}}\right] .
\end{aligned}
$$

Here $k_{d}$ is the diffuse wave number. To obtain the total density and current, we must add the reduced components to $u_{d}$ and $J_{d}$. In the case considered, $u_{r}=J_{r}=W \exp (-\bar{\mu} z)$ so that

$$
\begin{aligned}
& \frac{1}{W} u(z)=A e^{-\bar{\mu} z}+B e^{-k_{d} z}, \\
& \frac{1}{W} J(z)=\frac{\mu_{a}}{\bar{\mu}} A e^{-\bar{\mu} z}+\frac{\mu_{a}}{k_{d}} B e^{-k_{d} z} .
\end{aligned}
$$

This solution can be compared to the density and current obtained by solving the RTE without any approximations. Although one-dimensional RTE can be solved by a variety of analytical methods, we have used in this paper Monte Carlo simulations to obtain such solutions. The corresponding computational package has been developed by us and is publicly available [? ]. Further, we have used the nonlinear optimization algorithm implemented in Gnuplot to fit the analytical solution given in Eq. (26a) (for the density) to the Monte Carlo numerical solution. The variables $\bar{\mu}$ and $\ell$ have been used as the adjustable parameters in the fitting procedure. Results are shown in the figures below.

Simulations illustrated in Fig. 1 were performed for a medium with the ratio $\mu_{a} / \mu_{s}=0.03 / 500$ and Henyey-Greenstein phase function with the scattering asymmetry parameter $g=0.98$. These parameters are characteristic of biological soft tissues in the near-IR spectral range. We plot the total density in Panel (a), the total current in Panel (b) and the diffuse component of the current in Panel (c) for various values of the adjustable parameters. Different curves and data points shown in the figure are explained next.

The dots labeled MC in Fig. 1 are the result of Monte Carlo simulations. Note that, in order not to overcrowd the figure, we show only a few Monte Carlo data points in the plot. The total number of computed data points is 400 ; they are equally spaced between $z=0$ and $z=10 \ell^{*}$, and the Monte Carlo process was run to achieve high statistical confidence of each data point. All 400 data points were used in the optimization procedure. Note also that the Monte Carlo simulation was carried out in a much wider slab (either $50 \ell^{*}$ or $100 \ell^{*}$ wide), so that the effect of the far face of the slab is negligible in the interval $0<z<$ $10 \ell^{*}$, which is displayed in the figure. Finally, the leftmost data point was computed by accumulating the statistics of photons crossing the surface while all other data points were computed by accumulating the statistics of photons visiting a given volume voxel (a thin infinite layer for the one-dimensional problem considered here). Both approaches are correct, but the specific intensity changes very fast in a thin boundary layer and it is difficult to achieve accurate results by accumulating volume statistics in this region.

The curves labeled $\bar{\mu}=\mu_{t}$ and $\bar{\mu}=\mu^{*}$ in Fig. 1 have been computed according to Eq. (26) for the above values of $\bar{\mu}$ and $\ell=$ $2 \ell^{*} / 3$. It can be seen that both curves coincide with the Monte Carlo data at sufficiently large depths but deviate significantly near the surface, the loss of precision being more significant for the $\bar{\mu}=\mu^{*}$ curve. The maximum relative error is about $30 \%$ in 

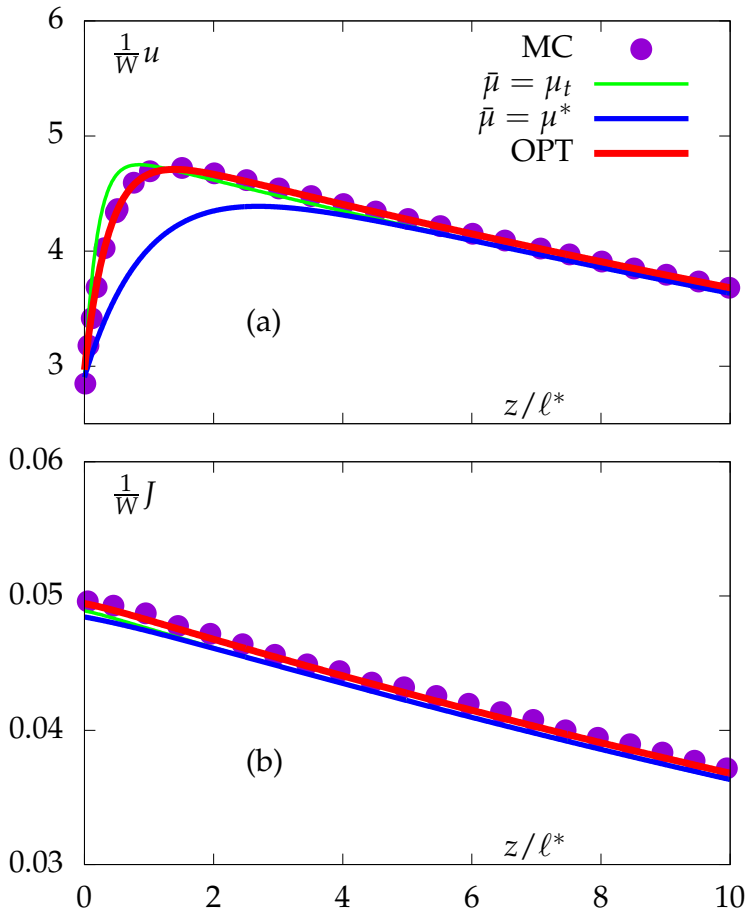

Fig. 2. Same as in Fig. 1 but for $g=0.8$ and the diffuse component of the current is not shown. The optimal parameters in this case are $\bar{\mu}_{\mathrm{opt}}=2.58 \mu^{*}, \ell_{\mathrm{opt}}=0.69 \ell^{*}$.

both cases, but the significant errors extent up to $z \sim 1 \ell^{*}$ for $\bar{\mu}=\mu_{t}$ and up to $z \sim 3 \ell^{*}$ for $\bar{\mu}=\mu^{*}$.

Finally, the curve labeled OPT was computed using the optimal values of $\bar{\mu}$ and $\ell, \bar{\mu}_{\text {opt }}=2.84 \mu^{*}$ and $\ell_{\text {opt }}=0.69 \ell^{*}$. This curve shows an excellent agreement with the MC data almost everywhere. The only exceptions are the points very close to the surface (separated from the surface by a small fraction of $\ell^{*}$ ), where any DA necessarily breaks. The cause of this breakdown is related not so much to the presence of continuous spectrum in the RTE solutions (which can not be captured in any diffusion theory) but rather the half-range boundary conditions of the RTE. We will illustrate the cause for this discrepancy when we consider the angular dependence of the specific intensity.

It is also interesting to note that the inaccuracy of the DA with suboptimal parameters affects mostly the density $u$ but not the current. The total current is almost independent of the choice of parameters and is always positive (directed into the medium) as could be expected from energy conservation. The diffuse component of the current, however, depends strongly on the parameters and is negative close to the surface. This can be easily understood: the negative current describes the diffuse reflectance of the medium.

In Fig. 2, we show the results for a similar medium but with $g=0.8$. In this case, the $\bar{\mu}=\mu_{t}$ curve is not dramatically inaccurate but the optimized curve is still much better. The optimal parameters for this medium are $\bar{\mu}_{\mathrm{opt}}=2.58 \mu^{*}, \ell_{\mathrm{opt}}=$ $0.69 \ell^{*}$. Generally, we have found that the ratio $\bar{\mu}_{\mathrm{opt}} / \mu^{*}$ tends to increase with $g$. For example, we have obtained the following values for this ratio: 1.43 for $g=0,1.96$ for $g=0.25,2.26$ for $g=$ 0.5 . Corresponding plots are not shown but in all cases excellent agreement has been obtained between the optimized curves and the Monte Carlo data points. The result $\bar{\mu}_{\text {opt }} / \mu^{*}=1.43$ (rather than unity) for $g=0$ should not be surprising. Recall that the RTE has a continuous and discrete spectra of eigenvalues $\lambda_{n}$ and that in the case of isotropic scattering there is only two discrete eigenvalues $\lambda_{d}= \pm 1 / k_{d}$ while the continuous spectrum is contained in the interval $-\mu_{t} \leq \lambda \leq \mu_{t}$. Also, $\mu_{t}=\mu^{*}=1 / \ell^{*}$ for isotropic scattering. The rate of exponential decay is the reciprocal of the eigenvalue $\lambda$, and the result $\bar{\lambda}_{\text {opt }}=1.43 \ell^{*}=$ $1.43 / \mu_{t}$ can be viewed as some weighed average of all exponents contained in the interval $\left[0,1 / \mu_{t}\right]$.

As for the ratio $\ell_{\mathrm{opt}} / \ell^{*}$, it remained relatively stable with a tendency to increase slightly with $g$, i.e., from 0.67 for $g=$ 0 to 0.69 for $g=0.98$. This behavior is somewhat counterintuitive. Indeed, the ratio $\ell_{\mathrm{opt}} / \ell^{*} \approx 0.71$ can be obtained from the exact analytical solution to the RTE for reflection from a halfspace with isotropic scattering and $\mu_{a} / \mu_{s} \rightarrow 0$ [26], which, in the case of the Henyey-Greenstein phase function, corresponds to $g=0$ (the Milne problem). We, however, obtain a similar ratio of 0.69 for a highly anisotropic forward-peaked scattering. Moreover, when we decrease $g$, the optimal ratio $\ell_{\text {opt }} / \ell^{*}$ does not tend to 0.71 but decreases and approaches $2 / 3$. In fact, the extrapolation distance of the exact solution to the Milne problem is not necessarily the optimal extrapolation distance for the DA; the two solutions are substantially different. It is also worth noting that adjusting the value of $\ell$ has only a minor effect on the fit quality; which is mainly influenced by the choice of $\bar{\mu}$.

In Fig. 3 we show results for a medium similar to that of Fig. 1 (with $g=0.98$ ) but with twice larger absorption. The results are qualitatively the same as in Fig. 1 , with $\bar{\mu}_{\text {opt }}=2.82 \mu^{*}, \ell_{\text {opt }}=$ $0.69 \ell^{*}$. It appears that the choice of optimal parameters does not depend on absorption significantly as long as $\mu_{a} / \mu_{s}$ is small. The difference of the optimal ratio $\bar{\mu}_{\text {opt }} / \mu^{*}$ with Fig. 1 (2.82 vs. $2.84)$ can be due to numerical errors. We note in this respect that, in order to achieve stably reproducible results, optimization must be performed in sufficiently large intervals of $z$. In our simulations, the optimized parameters were stable within $1 \%$ to $2 \%$ relative error and could depend within these limits on the particular realization of the Monte Carlo process and sampling of the data points.

It is now evident that the most challenging case for building any diffusion theory is the one with the largest ratio $\mu_{t} / \mu^{*}$. For the medium considered in Fig. 1 , this ratio is $\approx 50$. The optimized DA can still be very accurate in this case, but the correct choice of the reduced extinction coefficient $\bar{\mu}$ is important. We therefore use the medium of Fig. 1 to illustrate the quality of the conventional DA described in Sec. 3. Recall that the latter is obtained by solving Eq. (8) in which we set $S=E$ (or $\mathbf{Q}=0$ ) subject to the homogeneous boundary condition Eq. (14). For the one-dimensional problem considered here, this is mathematically equivalent to replacing the right-hand side of Eq. (22b) by zero; everything else remains unchanged. The resulting solution is still of the form Eq. (26) but the constants $A$ and $B$ are now different, viz,

$$
A=\frac{\bar{\mu}^{2}-3 \mu^{*} \bar{\mu}}{\bar{\mu}^{2}-k_{d}^{2}}, \quad B=\frac{3 \mu^{*} \bar{\mu}-k_{d}^{2}}{\bar{\mu}^{2}-k_{d}^{2}} \frac{1+\ell \bar{\mu}}{1+\ell k_{d}} .
$$

The solutions obtained according to the conventional DA are illustrated in Fig. 4. The curves labeled $\bar{\mu}=\mu^{*}$ in Fig. 4 and Fig. 1 are identical. The curve labeled $\bar{\mu}=\mu_{t}$, however, does not provide a reasonable approximation in the conventional DA (Fig. 4). The large-depth asymptote is in this case off by an overall factor but, of course, it has the same rate of exponential 

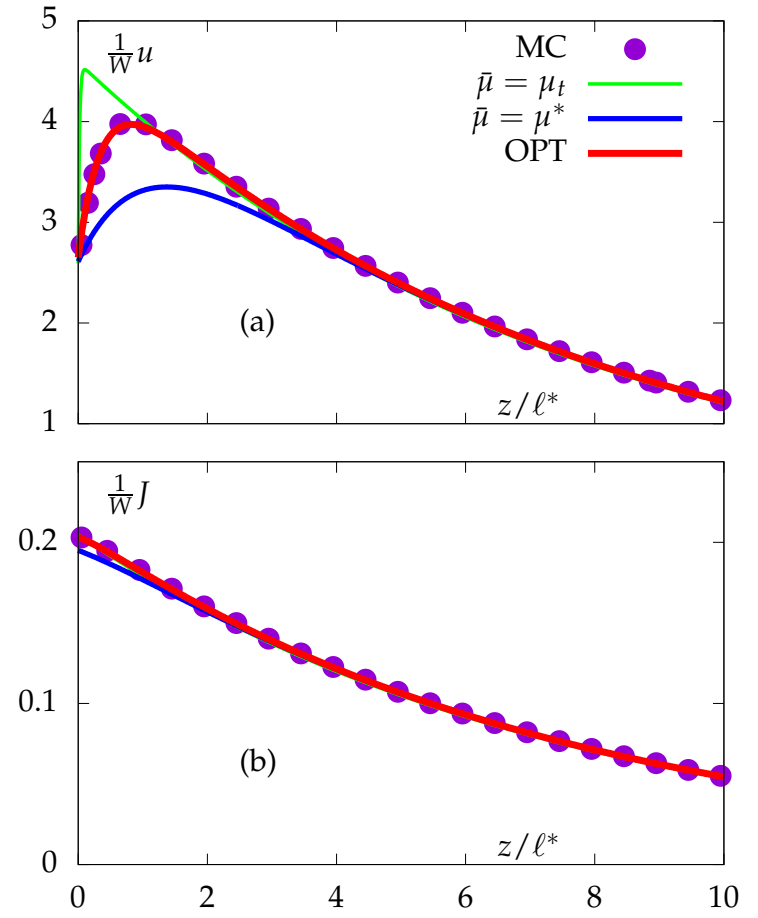

Fig. 3. Same as in Fig. 1 but for twice stronger absorption, $\mu_{a} / \mu_{s}=0.06 / 500$, and the diffuse component of the current is not shown. The optimal parameters in this case are $\bar{\mu}_{\text {opt }}=$ $2.82 \mu^{*}, \ell_{\mathrm{opt}}=0.69 \ell^{*}$.

decay described by the function $\exp \left(-k_{d} z\right)$. Near the boundary, the conventional DA can not be made accurate even by using optimization. The optimal parameters are in this case $\bar{\mu}_{\mathrm{opt}}=$ $1.31 \mu^{*}, \ell_{\text {opt }}=0.99 \ell^{*}$ and these parameters do not provide an accurate fit. The above optimal value of $\ell$ is quite far from the commonly accepted values (from $2 / 3$ to 0.71 ), which is an indication that the formula itself is wrong. The mismatch at the boundary persists in the case of a finite slab as well (data not shown), which is not the case for the optimized DA whose accuracy in finite slabs is illustrated next.

The optimized DA solutions in finite slabs are of a more general form than Eq. (24) or Eq. (26). Namely, we have in this case for the total current and density

$$
\begin{aligned}
& \frac{1}{W} u(z)=A e^{-\bar{\mu} z}+B_{1} e^{-k_{d} z}+B_{2} e^{k_{d} z} \\
& \frac{1}{W} J(z)=\frac{\mu_{a}}{\bar{\mu}} A e^{-\bar{\mu} z}+\frac{\mu_{a}}{k_{d}}\left(B_{1} e^{-k_{d} z}-B_{2} e^{-k_{d} z}\right) .
\end{aligned}
$$

Here the coefficient $A$ is the same as in Eq. (25a) but $B_{1}$ and $B_{2}$ are rather complicated. The corresponding expressions can be simplified by neglecting the terms that are exponentially small and of the order of $\exp (-\bar{\mu} L)$. These, typically, very small terms originate from the overlap of the reduced intensity with the far surface of the slab, which gives rise to inhomogeneous boundary conditions at that surface. Then $B_{1}$ and $B_{2}$ take the form

$$
\begin{aligned}
B_{1} & =\frac{\left(1+k_{d} \ell\right)^{2}}{\left(1+k_{d} \ell\right)^{2}-\left(1-k_{d} \ell\right)^{2} \exp \left(-2 k_{d} L\right)} B, \\
B_{2} & =\frac{\left(1-k_{d} \ell\right)\left[k_{d}^{2}\left(1-2 \ell \bar{\mu}+3 \ell \mu^{*}\right)-3 \bar{\mu}^{2}\left(1+\ell \mu^{*}\right)\right]}{\left(\bar{\mu}^{2}-k_{d}^{2}\right)\left[\left(1+k_{d} \ell\right)^{2} \exp \left(2 k_{d} L\right)-\left(1-k_{d} \ell\right)^{2}\right]},
\end{aligned}
$$
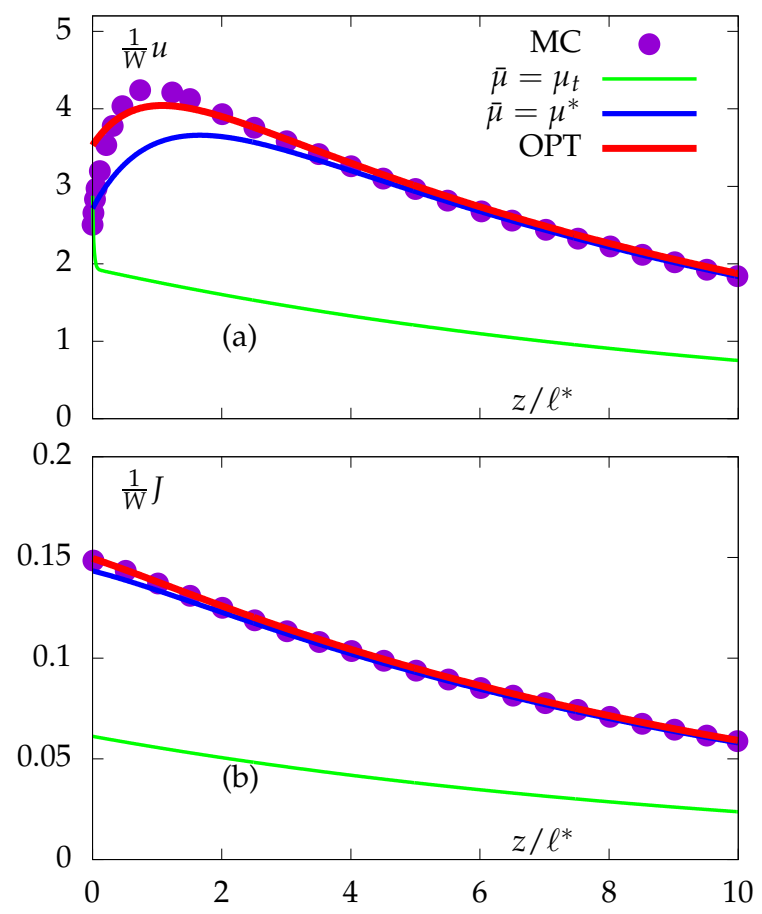

Fig. 4. Same as in Fig. 1 and for the same medium parameters, but analytical curves were computed according to the conventional DA. The optimal parameters in this case are $\bar{\mu}_{\text {opt }}=1.31 \mu^{*}, \ell_{\text {opt }}=0.99 \ell^{*}$. The curves $\bar{\mu}=\mu^{*}$ in this figure and in Fig. 1 are identical.

where $B$ is the constant defined in Eq. (25b).

In Fig. 5, we plot the total density according to the optimized DA in finite slabs of different widths $L\left(L=10,20,40 \ell^{*}\right)$ for a medium of the same optical properties as in Fig. 1. As before, the Monte Carlo statistics was accumulated in 400 thin layers and only approximately $1 / 20$ of all data points are shown in the figure. In addition, the left-most and the right-most data points were computed by counting the photons that cross the corresponding surface. It can be seen that the agreement is in all cases excellent. The slight discrepancy seen very close to either boundary in the case $L=10 \ell^{*}$ is of the same magnitude and extent as is seen in Fig. 1 near the boundary $z=0$ (about $10 \%$ relative error; errors of the same order of magnitude can be seen in the back-reflected quantities discussed in section 7 below for a pencil beam illumination). It should be emphasized that the optimized parameters used to compute the analytical curves in Fig. 5 were computed by considering the half-space data.

We next turn to the angular dependence of the intensity. Due to the azimuthal symmetry of the problem, it depends only on the polar angle $\theta$. In Fig. 6, we plot $I$ as a function of $\cos \theta$ at different values of $z$ near the interface $z=0$ through which the incident radiation enters the medium. The theoretical curves were computed according to Eq. (4) to which a term of the form $\exp \left(-\bar{\mu}_{\text {opt }} z\right) \delta_{\lambda}(\cos \theta)$ has been added, where

$$
\delta_{\lambda}(x)=\frac{\lambda}{\pi^{2}} \frac{1}{(x-1)^{2}+\lambda^{2}}
$$

is a forward-peaked angular delta function. The normalization factor in Eq. (30) is chosen so that $2 \pi \int_{-1}^{1} \delta_{\lambda}(x) d x \approx 1$. In Fig. 6 , 

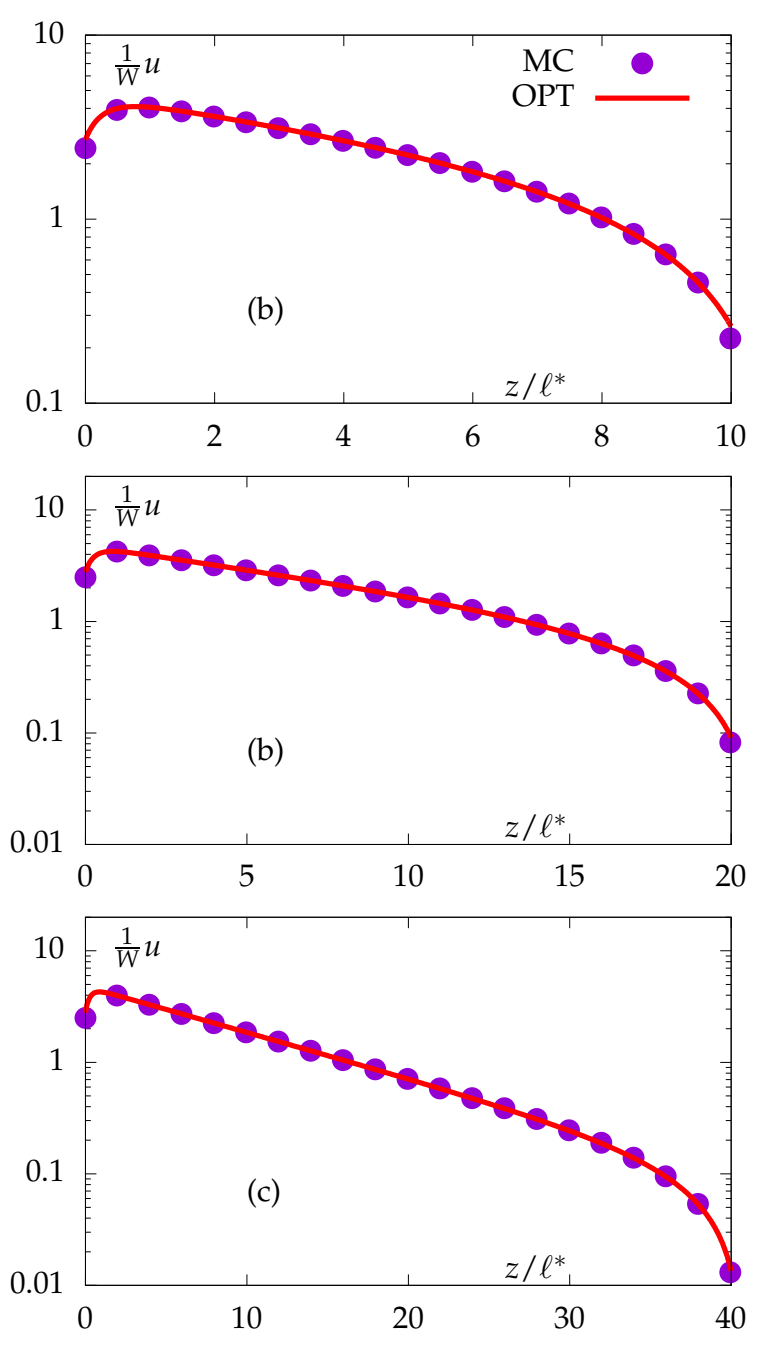

Fig. 5. Total density in finite slabs of of varying width for the same medium as in Fig. 1. Monte Carlo simulations vs optimized DA. The curves labeled OPT have the same parameters $\bar{\mu}_{\text {opt }}$ and $\ell_{\text {opt }}$ as in Fig. 1 . The total width of the slab corresponds to the interval of $z$ shown in each plot.

we have taken (quite arbitrarily) $\lambda=0.005$. The additional term defined above represents the reduced intensity. It is shown as a finite-width Lorentzian because a true delta-function can not be displayed in a plot. Even though this may be not obvious from the plots, the integral weight of this additional term is approximately equal to the integral weight of the forward peak that is seen in all Monte Carlo simulation results.

The curve labeled BR in Fig. 6(a) is the angular distribution of the back-reflected intensity. It was computed by a Monte Carlo simulation counting the outgoing photons that cross the $z=0$ boundary from inside the medium. The same function can also be defined analytically as a solution to a nonlinear integral equation and is known as the Chandrasekhar function [27]. For our purposes, the numerical Monte Carlo result suffices. It can be seen that the reflected intensity satisfies the half-range boundary condition of the RTE and is therefore zero for $\cos \theta>0$ (for all ingoing directions). Due to this reason, the shape of the curve is highly nonlinear and it is clear that this dependence can not be captured with any precision by a DA.
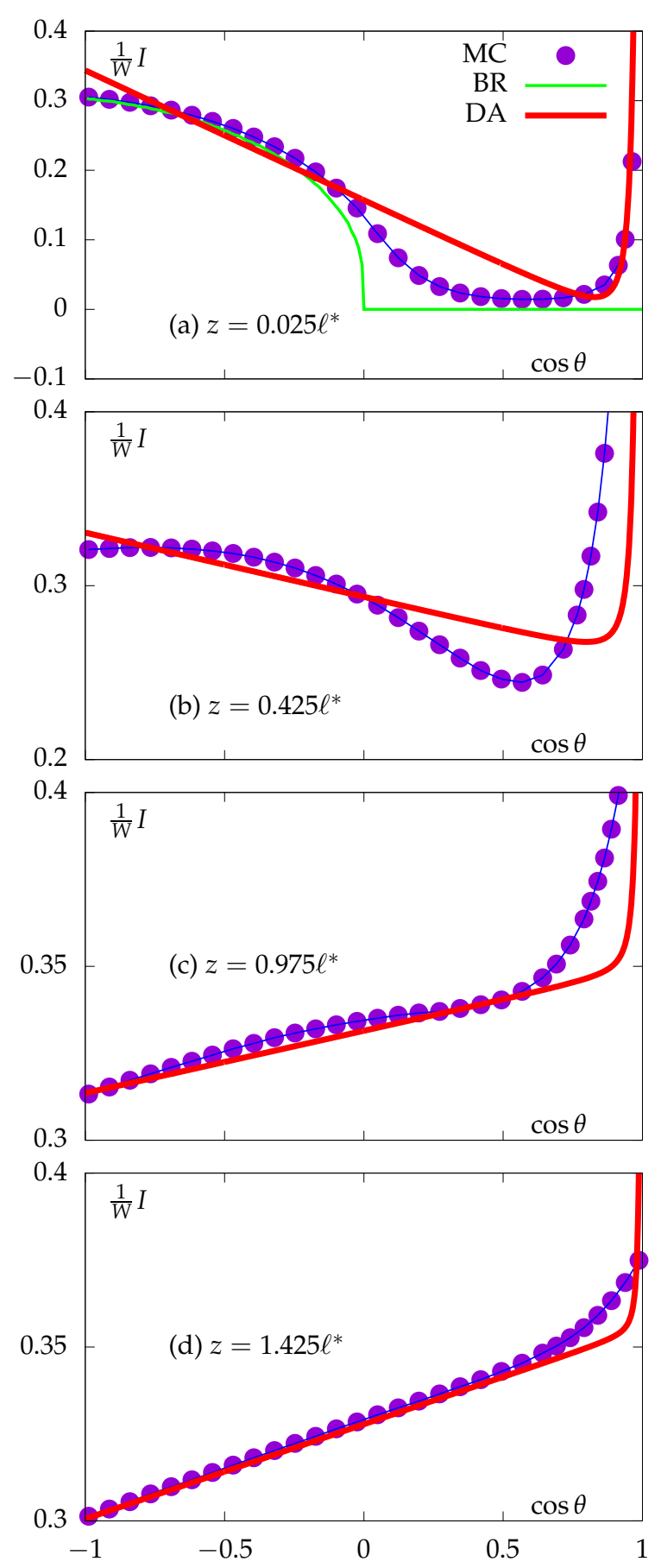

Fig. 6. Angular dependence of the intensity $I(z, \theta)$ at various depths $z$ as labeled for the same medium parameters as in Fig. 1. Monte Carlo simulations (MC) compared to the diffusion approximation (DA). Panel (a) also shows the angular dependence of the intensity back-reflected at the plane $z=0$ (BR). Not all MC data points are shown and some of them lie outside of the areas of the plots. The thin blue lines that connect the MC data points are drawn to guide the eye.

However, as soon as we evaluate $I(\theta)$ inside the medium, the shape of the curve starts to change. At $z=0.025 \ell^{*}$ (Fig. 6(a)), the intensity becomes nonzero for ingoing directions and it has 
a pronounced forward peak. The DA still can not capture this dependence accurately but some qualitative correspondence is obvious. At larger optical depths (Figs. 6(b-d)), the exact function becomes increasingly linear and the forward peak less pronounced. At $z=1.425 \ell^{*}$, the DA is already quite accurate. Of course, the DA can not predict accurately the width or exact shape of the forward peak, which is still present at this depth. It simply replaces it by a delta-function of approximately the same integral weight.

It can be concluded that any DA has three adjustable parameters: two parameters describing the linear segment of the angular dependence of the intensity and one parameter describing the evolution (exponential decay) of the forward peak. This last parameter is the reduced extinction coefficient $\bar{\mu}$, and it has not been considered in the traditional theory. In this paper, we have used a numerical optimization technique to find the optimal value of this parameter.

Another conclusion is that any DA can not be equally accurate for arbitrary angles of incidence. This follows from the fact that the angular distribution of the reflected intensity is not captured correctly by the DA and then using reciprocity relations for the Green's function of the RTE. The optimization performed in this paper applies to normal incidence only and it can be expected to remain accurate for incidence angles such that the curve labeled BR in 6(a) does not deviate too much from a linear behavior. In Sec. 5, we will show that this is indeed the case.

\section{NUMERICAL EXAMPLES FOR ONE-DIMENSIONAL PROPAGATION AND OBLIQUE INCIDENCE}

As was demonstrated above, the angular distribution of the back-reflected light is not well described by the DA. Invoking the reciprocity relation for the RTE Green's function, we can conclude that the optimization of $\bar{\mu}$ and $\ell$ that was performed above is not applicable to all incidence angles. Rather, it applies to normal incidence only. There is no reason to believe that the same optimized parameters would yield accurate approximation for oblique incidence.

Consider incident intensity that, in the half-space $z<0$, is of the form $I_{\text {inc }}=W \delta_{2}\left(\hat{\mathbf{s}}, \hat{\mathbf{s}}_{0}\right)$, where $\hat{\mathbf{s}}_{0}$ is some direction, generally, different from the direction of the $Z$-axis and making an angle $\theta$ with the latter. It can be shown that the surface source term for the RTE is in this case $\varepsilon(\mathbf{r}, \hat{\mathbf{s}})=W \cos \theta \delta(z) \delta_{2}\left(\hat{\mathbf{s}}, \hat{\mathbf{s}}_{0}\right)$. We can find the reduced intensity from Eq. (3). A straightforward calculation results in $I_{r}(z, \hat{\mathbf{s}})=W \delta_{2}\left(\hat{\mathbf{s}}_{,}, \hat{\mathbf{s}}_{0}\right) \exp (-\bar{\mu} z / \cos \theta)$, and the corresponding reduced density and current are $u_{r}(z)=$ $W \exp (-\bar{\mu} z / \cos \theta)$ and $\mathbf{J}_{r}(z)=W \hat{\mathbf{s}}_{0} \exp (-\bar{\mu} z / \cos \theta)$. For the diffuse components, we then have two coupled equations

$$
\begin{aligned}
& \hat{\mathbf{z}} \cdot \frac{\partial \mathbf{J}_{d}}{\partial z}+\mu_{a} u_{d}=W\left(\bar{\mu}-\mu_{a}\right) \exp (-\bar{\mu} z / \cos \theta), \\
& \frac{\hat{\mathbf{z}}}{3} \frac{\partial u_{d}}{\partial z}+\mu^{*} \mathbf{J}_{d}=W \hat{\mathbf{s}}_{0}\left(\bar{\mu}-\mu^{*}\right) \exp (-\bar{\mu} z / \cos \theta) .
\end{aligned}
$$

An important difference with the normal incidence case is that now $\mathbf{J}_{d}$ and $\mathbf{J}_{r}$ have more than one nonzero Cartesian components. Generally, we can assume that the vector $\hat{\mathbf{s}}_{0}$ lies in the $X Z$-plane and the same is true for $\mathbf{J}_{r}$ and $\mathbf{J}_{d}$. The boundary condition at the $z=0$ interface is

$$
u_{d}(0)+3 \frac{\ell}{\ell^{*}} J_{d z}(0)=0 .
$$

The solutions in the half-space $z>0$ for the total density and the normal component of the total current are

$$
\begin{aligned}
& \frac{1}{W} u(z)=A e^{-\bar{\mu} z / \cos \theta}+B e^{-k_{d} z}, \\
& \frac{1}{W} J_{z}(z)=\frac{\mu_{a}}{\bar{\mu}} A e^{-\bar{\mu} z / \cos \theta}+\frac{\mu_{a}}{k_{d}} B e^{-k_{d} z},
\end{aligned}
$$

where

$$
\begin{gathered}
A=-\frac{\left(3-1 / \cos ^{2} \theta\right) \bar{\mu}^{2}}{(\bar{\mu} / \cos \theta)^{2}-k_{d}^{2}}, \\
B=\frac{3 \bar{\mu}^{2}-k_{d}^{2}}{(\bar{\mu} / \cos \theta)^{2}-k_{d}^{2}}-\frac{k_{d} \ell}{1+k_{d} \ell} \frac{3 \bar{\mu} \cos \theta+k_{d}}{\bar{\mu} / \cos \theta+k_{d}} \\
\quad+\frac{3 \mu^{*} \ell \cos \theta}{1+k_{d} \ell} .
\end{gathered}
$$

In the case $\theta=0$ (normal incidence), these expressions coincide with those given in Eq. (25).

In Fig. 7, we plot Monte Carlo and theoretical (that is, given by Eq. (33)) results for the total density $u(z)$ and same medium parameters as in Fig. 1. As above, theoretical curves are plotted for various values of parameters where the optimal parameters were computed for the normal incidence. It can be seen that the optimized DA works reasonably well for moderate incidence angles, up to $\tan \theta \sim 0.5$, but breaks down for larger values of $\theta$. For $\tan \theta=2$, the DA results are not accurate even in the asymptotic regime. We note that the exact function $u(z)$ always has a maximum, albeit it can be very close to the surface for large incidence angles. The theoretical curves also have maxima for sufficiently small values of $\tan \theta$ but for $\tan \theta$ larger than some critical value, the theoretical function are monotonically decreasing. Overall, it can be concluded that, for $\tan \theta \gtrsim 1$, the standard and the optimized DAs are all inaccurate.

Another interesting feature of oblique incidence, which is not captured quantitatively by any DA is the lateral current. As mentioned above, the current $\mathbf{J}$ has two nonzero Cartesian components, which lie in the plane $X Z$ spanned by the vector $\hat{\mathbf{s}}_{0}$ and the Z-axis. In Eq. (33b), only the normal $z$-component of the current was given. The lateral component $J_{x}$ (the total current) can also be found from Eq. (31) and is given by

$$
\frac{1}{W} J_{x}=\frac{\bar{\mu}}{\mu^{*}} \sin \theta \exp (-\bar{\mu} z / \cos \theta) .
$$

Interestingly, this current physically exists in the system even though $\partial u_{d} / \partial x=0$ and, thus, this current is not subject to Fick's law. The lateral current, however, decays exponentially with the depth $z$. The lateral current is plotted in Fig. 8. It can be seen that the theoretical formula Eq. (35) describes this current qualitatively but not quantitatively. Indeed, the lateral current is not a diffusion phenomenon and can be properly accounted for only by the transport theory.

\section{EFFECTS OF THE PHASE FUNCTION}

So far, we have considered only the Henyey-Greenstein phase function, that is, we assumed that

$$
A\left(\hat{\mathbf{s}}, \hat{\mathbf{s}}^{\prime}\right)=\frac{1}{4 \pi} \frac{1-g^{2}}{\left(1-2 g \hat{\mathbf{s}} \cdot \hat{\mathbf{s}}^{\prime}+g^{2}\right)^{3 / 2}},
$$

where $g$ is scattering asymmetry parameter. Although $g$ defines the Henyey-Greenstein functions completely, a more general 

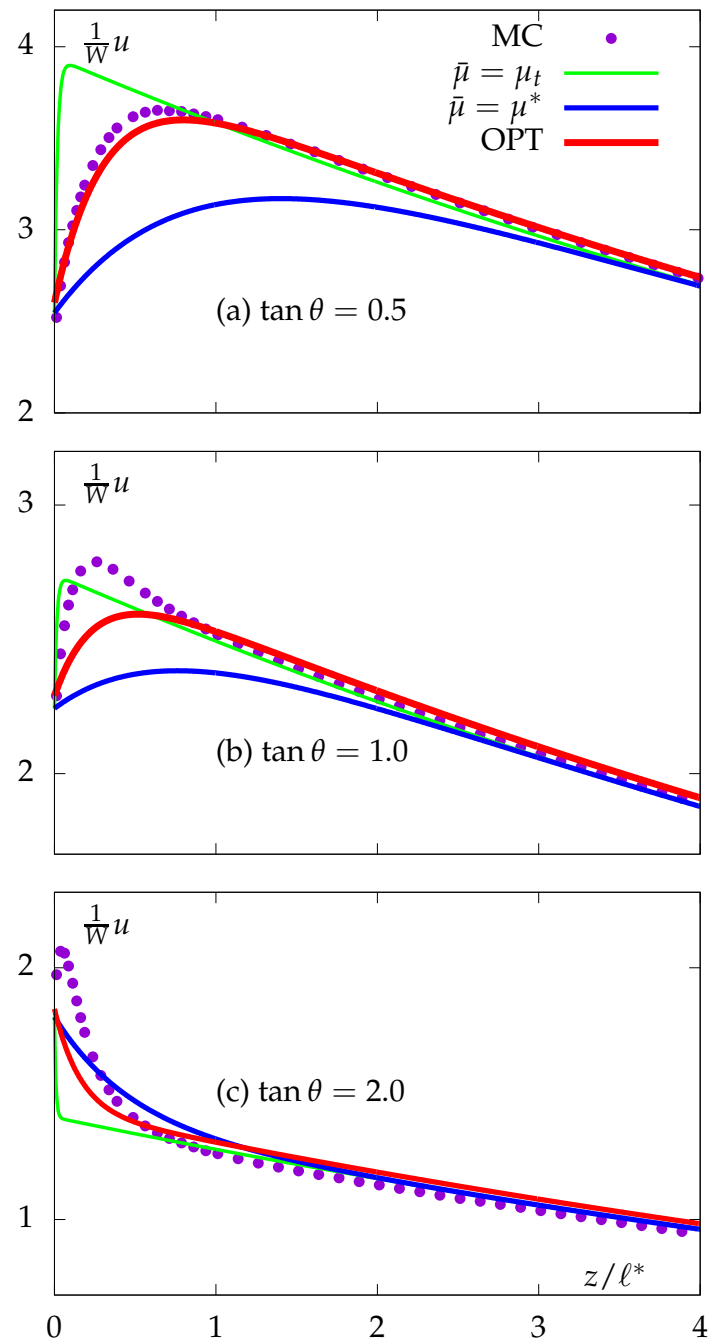

Fig. 7. Same as in Fig. 1 but for off-normal incidence. Here $\theta$ is the angle between the collimation direction of incident radiation and normal to the surface; $\theta=0$ for normal incidence. However, the front of the incident radiation is still infinitely broad and the problem is one-dimensional.

phase function is not defined by $g$. In other words, there are many different phase functions with the same value of $g$. For example, we can consider an exponential function of the form

$$
A\left(\hat{\mathbf{s}}, \hat{\mathbf{s}}^{\prime}\right)=\frac{\beta}{4 \pi} \frac{\exp \left(\beta \hat{\mathbf{s}} \cdot \hat{\mathbf{s}}^{\prime}\right)}{\sinh (\beta)} .
$$

Here the scattering asymmetry parameter is related to $\beta$ by $g=\operatorname{coth}(\beta)-1 / \beta$. This equation can be uniquely inverted, and for every $0<g<1$ we can find the corresponding parameter $\beta$. It should be noted that Henyey-Greenstein and exponential phase functions with the same $g$ are quite different. In particular, the probability of backscattering is much smaller in the later than in the former case.

In Fig. 9, we plot the total density as a function of depth $z$ for one-dimensional propagation in a finite slab of the width $L=10 \ell^{*}$. The theoretical solutions are given in this case by Eq. (28). We have used two different phase functions (HenyeyGreenstein and exponential) with the same value of $g$ in Monte Carlo simulations. It can be seen that the numerical solutions
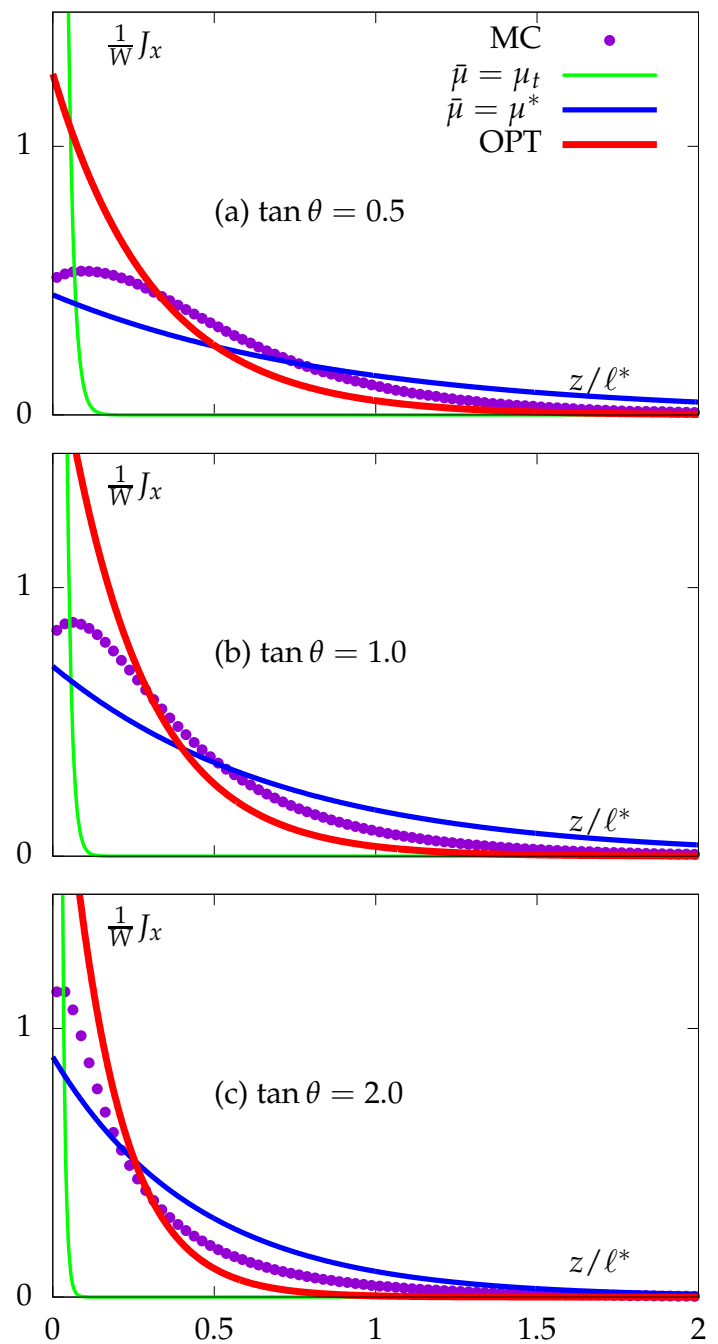

Fig. 8. Lateral current $J_{x}$ for off-normal incidence. Same medium and simulation parameters as in Fig. 7.

for these two phase functions differ significantly for $z \lesssim 2 \ell^{*}$. However, in the asymptotic region, and near the far face of the slab, the solutions essentially coincide. This is an expected result: the higher angular moments of the phase function are important only sufficiently close to the source.

However, the goal of this paper is to find a DA that is as accurate as possible everywhere, including close to the source. It can be seen that no such universal approximation exists. The optimal parameters depend on the higher moments of the phase function. For the exponential phase function the optimal values are $\bar{\mu}_{\text {opt }}=4.38 \mu^{*}$ and $\ell_{\text {opt }}=0.71 \ell^{*}$, which differs from the respective values for the Henyey-Greenstein function $\left(\bar{\mu}_{\mathrm{opt}}=\right.$ $2.84 \mu^{*}$ and $\ell_{\mathrm{opt}}=0.69 \ell^{*}$. The parameter that is changed most is $\bar{\mu}_{\text {opt }}$ but $\ell_{\text {opt }}$ is also noticeably different.

It is interesting to note that $\ell_{\text {opt }}$ for the exponential phase function matches closely the theoretical result deduced from the solution of the Milne problem for isotropic scattering. Also, the quality of the fit is better for the Henyey-Greenstein phase function. This is probably due to the fact that the exponential phase function suppresses strongly backscattering and, as a result, the diffuse regime is reached after more scattering events. 


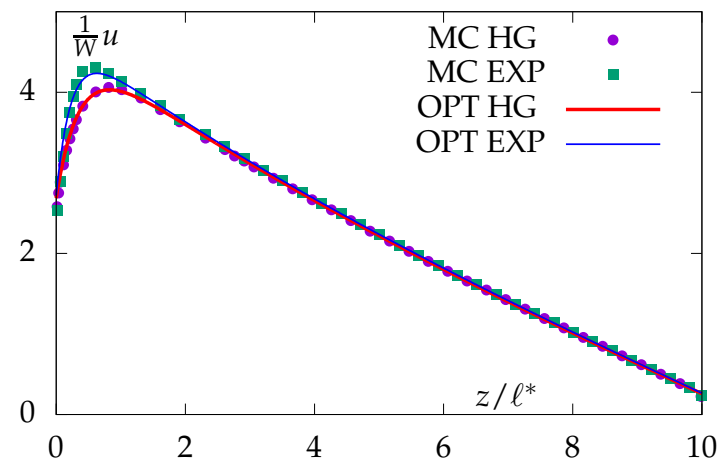

Fig. 9. Density as a function of depth $z$ for the same medium parameters as in Fig. 1 but two different phase functions labeled as HG (Henyey-Greenstein) and EXP (exponential). MC and OPT label Monte Carlo simulations and optimized theoretical curves computed according to Eq. (28). Simulations were performed in a finite slab of width $L=10 \ell^{*}$. Optimal parameters are different for the HG and OPT curves. For HG, the parameters are the same as in Fig. 1. For EXP, $\bar{\mu}_{\mathrm{opt}}=4.38 \mu^{*}$, $\ell_{\mathrm{opt}}=0.71 \ell^{*}$

\section{NUMERICAL EXAMPLES IN THREE DIMENSIONS}

Consideration of a narrow collimated incident beam is somewhat complicated by the following fact. If we take the incident beam to be described mathematically by a delta-function in the transverse directions, i.e., $\delta(\rho)$, where $\rho=(x, y)$, then the solutions to both the RTE and the DE will contain singularities, which are difficult to represent or compare graphically. We therefore will consider an incident beam of small but finite width. Let the RTE source function be of the form

$$
\varepsilon(\boldsymbol{\rho}, z)=w(\boldsymbol{\rho}) \delta(z) \delta_{2}(\mathbf{s}, \hat{\mathbf{z}}), \quad \int w(\boldsymbol{\rho}) d^{2} \rho=W .
$$

Here $W$ is the total power of the incident beam (incoming energy per unit time, but not per unit surface, as was the case in the onedimensional geometry). We assume that the beam is perfectly collimated and all incident rays are parallel to the $Z$-axis. This is expressed mathematically by the angular delta-function $\delta_{2}(\mathbf{s}, \hat{\mathbf{z}})$ in Eq. (38). The reduced intensity is given in this case by

$$
I_{r}(\boldsymbol{\rho}, z)=w(\boldsymbol{\rho}) e^{-\bar{\mu} z} \delta_{2}(\mathbf{s}, \hat{\mathbf{z}}) .
$$

Correspondingly, the source functions in Eq. (6) are

$$
E(\boldsymbol{\rho}, z)=w(\boldsymbol{\rho}) e^{-\bar{\mu} z}, \mathbf{Q}(\boldsymbol{\rho}, z)=\hat{\mathbf{z}} E(\boldsymbol{\rho}, z) .
$$

We can now solve Eq. (6) by Fourier transform. To this end, we use the integral expansion

$$
u_{d}(\boldsymbol{\rho}, z)=\int \tilde{u}_{d}(\mathbf{q}, z) e^{i \mathbf{q} \cdot \boldsymbol{\rho}} \frac{d^{2} q}{(2 \pi)^{2}},
$$

and similarly for all other $\rho$-dependent functions. Upon substitution into Eq. (6), we find the following pair of equations for the Fourier transforms of the diffuse density $\tilde{u}_{d}(\mathbf{q}, z)$ and the $z$-component of the diffuse current $\tilde{J}_{d z}(\mathbf{q}, z)$ :

$$
\begin{aligned}
& \frac{\partial \tilde{J}_{d z}(\mathbf{q}, z)}{\partial z}+\left(\frac{q^{2}}{3 \mu^{*}}+\mu_{a}\right) \tilde{u}_{d}(\mathbf{q}, z)=\left(\bar{\mu}-\mu_{a}\right) e^{-\bar{\mu} z} \tilde{w}(q) \\
& \frac{1}{3} \frac{\partial \tilde{u}_{d}(\mathbf{q}, z)}{\partial z}+\mu^{*} \tilde{J}_{d z}(\mathbf{q}, z)=\left(\bar{\mu}-\mu^{*}\right) e^{-\bar{\mu} z} \tilde{w}(q)
\end{aligned}
$$

Once these equations are solved, the radial component of the diffuse current, $\mathbf{J}_{d \perp}$, can be found from the equation

$$
\tilde{\mathbf{J}}_{d \perp}(\mathbf{q}, z)=-\frac{i \mathbf{q}}{3 \mu^{*}} \tilde{u}_{d}(\mathbf{q}, z)
$$

The solution to Eq. (42) with the boundary condition (12) (which can also be Fourier transformed) is similar in form to Eq. (24) with a few modifications, namely,

$$
\begin{aligned}
& \tilde{u}_{d}(\mathbf{q}, z)=\left\{[A(q)-1] e^{-\bar{\mu} z}+B(q) e^{-\kappa(q) z}\right\} \tilde{w}(\mathbf{q}), \\
& \tilde{J}_{d z}(\mathbf{q}, z)=\left\{[C(q)-1] e^{-\bar{\mu} z}+\frac{\kappa(q)}{3 \mu^{*}} B(q) e^{-\kappa(q) z}\right\} \tilde{w}(\mathbf{q}) .
\end{aligned}
$$

where

$$
\begin{aligned}
& A(q)=-\frac{2 \bar{\mu}^{2}+q^{2}}{\bar{\mu}^{2}-\kappa^{2}(q)}, C(q)=-\frac{2 \mu_{a} \bar{\mu}+\frac{\bar{\mu}}{\mu^{*}} q^{2}}{\bar{\mu}^{2}-\kappa^{2}(q)}, \\
& B(q)=\frac{3 \bar{\mu}^{2}-\kappa^{2}(q)}{\bar{\mu}^{2}-k_{d}^{2}}+\frac{3 \ell}{1+\kappa(q) \ell}\left[\mu^{*}-\frac{\bar{\mu} \kappa(q)+\frac{k_{d}^{2}}{3}}{\bar{\mu}+\kappa(q)}\right], \\
& \kappa(q)=\sqrt{k_{d}^{2}+q^{2}}
\end{aligned}
$$

It can be seen that the expressions $(44),(45)$ are reduced to (24),(25) in the case $q=0$. As before, we need to add the diffuse and the reduced components of the density and current to obtain the total quantities. It follows from Eq. (39) that $\tilde{u}_{r}(\mathbf{q}, z)=e^{-\bar{\mu} z} \tilde{F}(\mathbf{q}), \tilde{\mathbf{J}}_{r}(\mathbf{q}, z)=\hat{\mathbf{z}} u_{r}(\mathbf{q}, z)$, so that the addition results in cancellation of the unities in the square brackets in Eq. (44). This is similar to the transition from Eq. (24) to Eq. (26); here we do not write out expressions for the total quantities explicitly.

We now specialize to the case of a symmetric pencil beam of radius $a$ described by the function $\tilde{w}(\mathbf{q})=\frac{2 W}{a q} J_{1}(a q)$, where $J_{1}(x)$ is the cylindrical Bessel function of the first kind (not to be confused with the current density). We can use the Fourier-space solution (44) and the transformation rule (41) to compute the solutions in real space. The Fourier integral can not be computed analytically but is easy to evaluate numerically. We note that the integral is converging and contains no singularities. In particular, the integrand does not have a singularity at $q^{2}=\bar{\mu}^{2}-k_{d}^{2}$, even though the coefficients $A(q), B(q)$ and $C(q)$ are singular at this point.

The radial dependence of the density $u(\rho, z)$ at different depth $z$ inside the medium is shown in Fig. 10. Parameters of the medium are the same as in Fig. 1 except that the incident field is now a pencil beam of the radius $0.5 \mu^{*}$. Although optimization of the reduced extinction coefficient $\bar{\mu}$ was carried out for an infinite-front, plane incident wave, we see that the optimized DA still outperforms the conventional DAs. In fact, it can be seen that the optimized DA is noticeably more accurate up to the depth of $z \sim 8 \ell^{*}$. We note that all DA predict a jump of the density at the edge of the incident pencil beam, that is, at $\rho=a$ while the exact solution is continuous everywhere. We note that the conventional DAs with $\bar{\mu}=\mu^{*}$ and $\bar{\mu}=\mu_{t}$ can be accurate for some values of the parameters, but the optimized DA is more universally applicable.

We next consider the density and current exactly at the interface, that is, at $z=0$. As was already shown in section 4 for wide-front plane-wave illumination, any DA is not capable of capturing correctly the angular dependence of the backscattered intensity, $I(\hat{\mathbf{s}})$. There is no reason why this conclusion would 

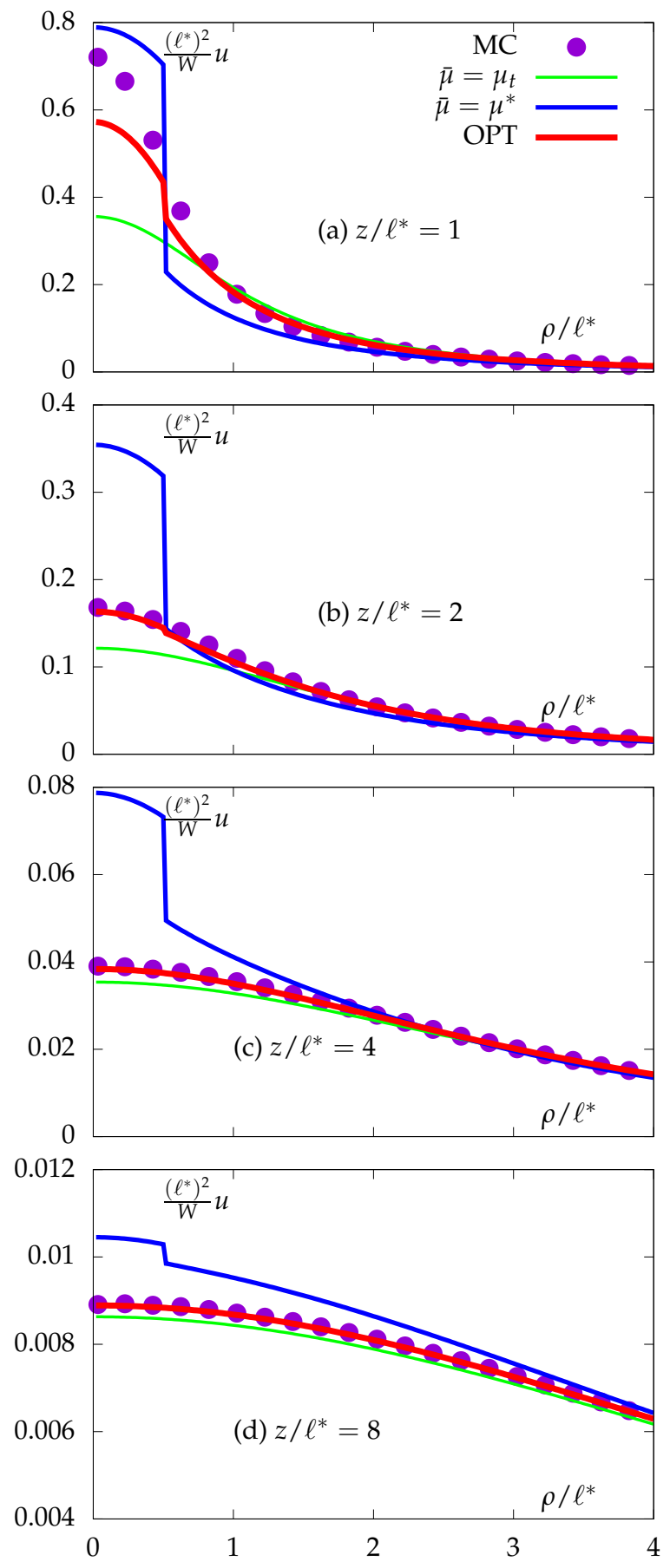

Fig. 10. Radial dependence of $u$ at different depths $z$ as labeled. Incident cylindrical pencil beam has the radius of $0.5 \ell^{*}$ (diameter of $1 \ell^{*}$ ). Labels of different curves and data sets are the same as in Fig. 1. Note that the discontinuity of solutions at the edge of the incident beam (at $\rho=0.5 \ell^{*}$ ) is an artifact of the DA in which the reduced (discontinuous) density is added to the diffuse (continuous) density; the exact RTE solutions are continuous, albeit the radial derivative can be large at the edge of the incident beam.

not be applicable to the three-dimensional geometry of a narrow incident beam, which is considered in this section. In fact, com-
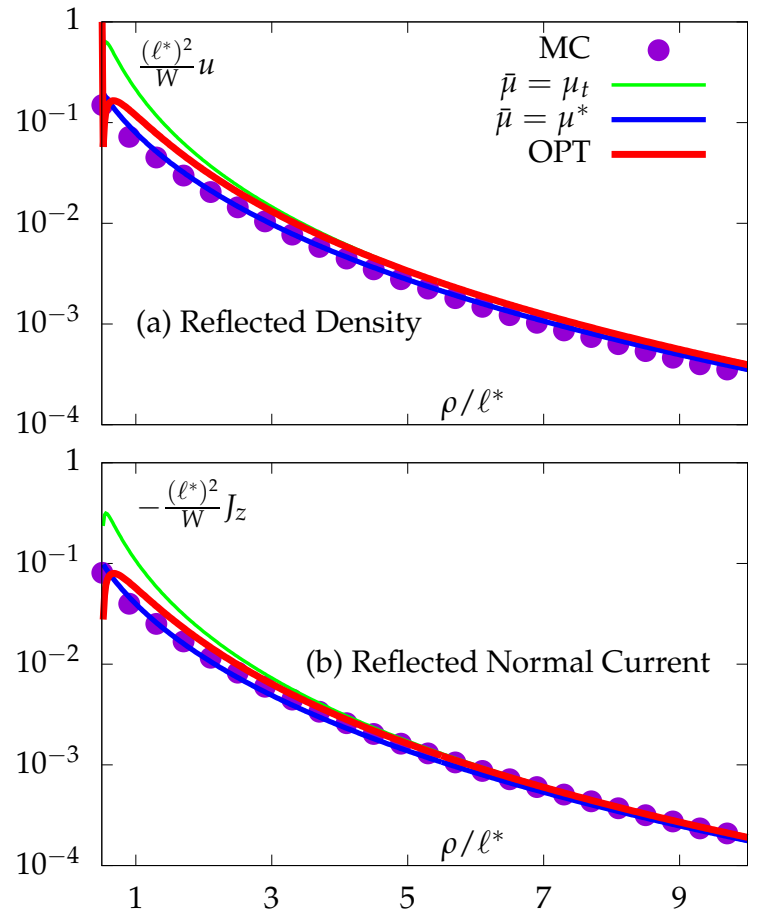

Fig. 11. Radial dependence of the reflected density $u$ (a) and the normal component of the current $J_{z}(\mathrm{~b})$, evaluated at the interface $z=0$. Same parameters as in Fig. 10.

parison of the quantities computed exactly at the interface by Monte Carlo simulations and from a DA is not very meaningful. Indeed, the DA always accounts for hypothetical photons that propagate in the incoming directions; while we know from the RTE half-range boundary conditions that the specific intensity is strictly zero in such directions (at the interface). Therefore, the predictions of a DA for, say, the normal component of current at the interface do not correspond to measurable quantities.

Nevertheless, we plot in Fig. 11 the density $u$ and the normal component of the current, $J_{z}$ at the interface. Again, the predictions of various DAs are compared to the rigorous Monte Carlo results. In this figure, the range of the radial variable $\rho$ is $a<\rho<10 \ell^{*}$, where $a=0.5 \ell^{*}$ is the radius of the incident pencil beam. Therefore, the region close to the axis of the beam $(\rho<a)$ is excluded from consideration. While the Monte Carlo results in this region are meaningful, the DA predictions can change sign and are, generally, meaningless. Besides, we note that it is technically difficult to measure the back-scattered intensity emitted exactly from the point where the incident beam enters the medium. This signal can be dominated by specular reflection, for example. However, as soon as we cross the point $\rho=a$, the agreement between the DAs and the Monte Carlo results becomes quite decent. It is interesting to note that, for the quantities displayed in Fig. 11, the DA with $\bar{\mu}=\mu^{*}$ provides the best approximation. The fact that $\bar{\mu}=\bar{\mu}_{\text {opt }}$ does not provide the best approximation in this case is not surprising. Indeed, we have performed the optimization of $\bar{\mu}$ for the density inside the medium, not on its boundary. We also note that, in the case of non-contact measurements, the quantity of interest is $I\left(\mathbf{r}_{d}, \hat{\mathbf{n}}\right)$, where $\mathbf{r}_{d}$ is a point on the surface corresponding to a given optical detector (say, a CCD pixel) and $\hat{\mathbf{n}}$ is the unit vector pointing 
from $\mathbf{r}_{d}$ to the detector (say, the optical axis of the CCD camera). In the case of contact measurements, some convolution of the form $\int F(\mathbf{s}) I\left(\mathbf{r}_{d}, \hat{\mathbf{s}}\right) d^{2} s$ is measured, where $F(\mathbf{s})$ is the angular acceptance function; the signal becomes equal to $J\left(\mathbf{r}_{d}\right)$ in the special case $F(\mathbf{s})=\hat{\mathbf{n}} \cdot \hat{\mathbf{s}}$.

\section{SUMMARY AND DISCUSSION}

We have discussed construction of the source function for the diffusion equation (DE), which serves as an approximation to a more fundamental radiative transport equation (RTE). We were particularly interested in the case when the incident radiation enters a multiply-scattering medium from outside through a diffuse-nondiffuse interface. The stationary DE can be written as two first order ordinary differential equations (6) for the diffuse density and current $u_{d}$ and $\mathbf{J}_{d}$. These equations contain two source terms $E(\mathbf{r})$ and $\mathbf{Q}(\mathbf{r})$. Alternatively, one can transform Eq. (6) into a second order equation (8). In this case, there is only one scalar source term $S(\mathbf{r})$, which is expressed in terms of $E(\mathbf{r})$ and $\mathbf{Q}(\mathbf{r})$ according to Eq. (9). The main goal of this paper was to find the optimal (that is, yielding the most accurate approximation) relation between the source function of the RTE $\varepsilon(\mathbf{r}, \hat{\mathbf{s}})$ and the function $S(\mathbf{r})$. The problem is nontrivial because the latter function has less degrees of freedom. The dependence of the RTE source term $\varepsilon(\mathbf{r}, \hat{\mathbf{s}})$ on $\hat{\mathbf{s}}$ is mimicked in the diffusion approximation (DA) by a more complicated dependence of $S(\mathbf{r})$ on $\mathbf{r}$.

In a DA, the total density and current of radiation energy in a multiply-scattering medium are given by sums of diffuse and reduced components, i.e., $u=u_{d}+u_{r}, \mathbf{J}=\mathbf{J}_{d}+\mathbf{J}_{r}$, where the reduced density and current are expressed in terms of the reduced intensity $I_{r}$ according to Eq. (7). However, the reduced intensity can be defined in infinitely many different ways. Each definition gives rise to a particular DA. Following [13], we have parameterized this family of DAs by the reduced extinction coefficient $\bar{\mu}$, which is the rate of exponential decay of the reduced intensity away from the source. It turns out that the source functions $E(\mathbf{r})$ and $\mathbf{Q}(\mathbf{r})$, or $S(\mathbf{r})$, are defined by $\bar{\mu}$ uniquely, so that the task is to find the optimal value of $\bar{\mu}$. We have approached this task by comparing the theoretical predictions of the DA to the results of Monte Carlo simulation and optimizing the discrepancy in terms of $\bar{\mu}$ and also in terms of the extrapolated distance parameter $\ell$. We have found that the optimal value of $\ell$ is close to the conventionally used value (between $2 \ell^{*} / 3$ and $0.71 \ell^{*}$ ), but our results for the optimal value of $\bar{\mu}$ are unconventional. The two conventional choices for the reduced extinction coefficient are $\bar{\mu}=\mu_{t}$ or $\bar{\mu}=\mu^{*}$. We however find that the optimal value of $\bar{\mu}$ lies between these two values; typically, $\bar{\mu}_{\text {opt }} \approx 3 \ell^{*} \ll \mu_{t}$. We note however that the numerical value of $\bar{\mu}_{\text {opt }}$ depends on the scattering phase function of the medium (even if the scattering asymmetry parameter is fixed) and, potentially, on the angle of incidence. We have performed optimization for normal incidence only.

There are good reasons why the reduced extinction coefficient $\bar{\mu}$ has not been considered previously. If the RTE source $\varepsilon(\mathbf{r}, \hat{\mathbf{s}})$ is localized inside the medium and far from the boundaries and if the point of observation is far from the support of $\varepsilon(\mathbf{r}, \hat{\mathbf{s}})$, then the exact shape of $S(\mathbf{r})$ is unimportant as long as the zeroth moment $M_{0}=\int S(\mathbf{r}) d^{3} r$ is approximately constant. As was shown in [13], $M_{0}$ depends on $\bar{\mu}$ weakly. This consideration applies to the definitions of $S(\mathbf{r})$ that were considered in Refs. [19-21]. If the source of the RTE is supported on the medium boundary (the so-called surface source), the next moment of $S(\mathbf{r}), \mathbf{M}_{1} \int \mathbf{r} S(\mathbf{r}) d^{3} r$, comes into play. However, the typical boundary conditions for the DA result in linear relations between $M_{0}$ and $\mathbf{M}_{1}$, e.g., Eq. (17), which also seems to indicate that the exact value of $\bar{\mu}$ is unimportant. From the above arguments, we can conclude the following. If the samples are a few centimeters thick and the region of interest is sufficiently far from the boundaries (as is the case in many implementations of optical tomography), the correct definition of $S(\mathbf{r})$ is not critical.

However, in the reflection geometry and, especially, when the region of interest is relatively shallow, the effects associated with an imprecise definition of $S(\mathbf{r})$ come to the fore. We emphasize that the function $S(\mathbf{r})$ depends strongly on $\bar{\mu}$. Correspondingly, the solutions in the superficial layers also depend strongly on $\bar{\mu}$, and this dependence is not reduced to multiplication by a constant. We have found that a simple optimization procedure can find an optimal value of $\bar{\mu}$, which can reduce the error quite dramatically close to the surface and not affect the accuracy far away from the surface. This result can be particularly useful in imaging modalities involving diffuse reflection measurements or when inhomogeneities of the medium can be located close to the interface.

The theory developed in this paper is, in fact, an attempt to account phenomenologically for the transition layers without abandoning the diffusion theory framework altogether. As was shown in [12], the solutions to the RTE can be approximated with high accuracy by a superposition of the interior and the boundary layer terms, where the former satisfies a DE. However, the boundary layer term can not be computed from a DE. If it is computed according to the procedure described in [12], an accurate approximation to the angular dependence of the intensity at the medium boundary can be obtained. Obviously, this dependence is highly nonlinear and can not be described in principle by a DA alone. The boundary layer solution can be added to the DA solution. In this paper, however, we took a different approach and stayed entirely withing the framework of the diffusion theory. We have shown that even in this case the boundary layers can be accounted for by appropriately splitting the total intensity into the reduced and diffuse parts. It can also be said that the reduced intensity is itself an additive term and that, by tuning the value of $\bar{\mu}$, we can select the optimal value of this additive term. This observation establishes a correspondence between the approaches of this paper and Ref. [12].

Finally, we have only considered stationary propagation in this paper. If time-dependent solutions are sought, one can consider additional adjustable parameters to improve accuracy at short times. It was suggested that this can be achieved by using the telegraph equation [28], which contains a second-order time derivative. In the stationary regime, the telegraph equation and the DE considered in this paper are equivalent. It is possible that combining the approach of this paper and using the telegraph equation as suggested in [28] can produce an accurate approximation at both short times and short distances.

\section{ACKNOWLEDGMENT}

This work has been supported by the French National Research Agency (ANR) under the Projects ANR-16-CE19-0012-04 and ANR-11-IDEX-0001-02.

\section{REFERENCES}

1. D. A. Boas, D. H. Brooks, E. L. Miller, C. A. DiMarzio, M. Kilmer, R. J. Gaudette, and Q. Zhang, "Imaging the body with diffuse optical tomography," IEEE Signal Proc. Mag. 18, 57-75 (2001). 
2. S. R. Arridge and J. C. Schotland, "Optical tomography: forward and inverse problems," Inverse Problems 25, 123010 (2009).

3. G. S. Abdoulaev and A. H. Hielscher, "Three-dimensional optical tomography with the equation of radiative transfer," J. Electron. Imag. 12, 594-601 (2003).

4. A. Joshi, J. C. Rasmussen, E. M. Sevick-Muraca, T. A. Wareing, and J. McGhee, "Radiative transport-based frequency-domain fluorescence tomography," Phys. Med. Biol. 53, 2069-2088 (2008).

5. G. Bal, "Inverse transport theory and applications," Inverse Problems 25, 053001 (2009).

6. A. Ishimaru, "Diffusion of a pulse in densely distributed scatterers," J. Opt. Soc. Am. A 68, 1045-1050 (1978).

7. A. D. Kim and A. Ishimaru, "Optical diffusion of continuous-wave, pulsed, and density waves in scattering media and comparisons with radiative transfer," Appl. Opt. 37, 5313-5319 (1998).

8. R. Aronson and N. Corngold, "Photon diffusion coefficient in an absorbing medium," J. Opt. Soc. Am. A 16, 1066-1071 (1999).

9. R. Elaloufi, R. Carminanti, and J.-J. Greffet, "Definition of the diffusion coefficient in scattering and absorbing media," J. Opt. Soc. Am. A 20, 678-685 (2003).

10. R. Pierrat, J.-J. Greffet, and R. Carminati, "Photon diffusion coefficient in scattering and absorbing media," J. Opt. Soc. Am. A 23, 1106-1110 (2006).

11. R. C. Haskell, L. O. Svaasand, T. T. Tsay, T. C. Feng, and M. S. McAdams, "Boundary-conditions for the diffusion equation in radiative transfer," J. Opt. Soc. Am. A 11, 2727-2741 (1994).

12. A. D. Kim, "Correcting the diffusion approximation at the boundary," J. Opt. Soc. Am. A 28, 1007-1015 (2011).

13. M. Machida, G. Y. Panasyuk, J. C. Schotland, and V. A. Markel, "Diffusion approximation revisited," J. Opt. Soc. Am. A 26, 1291-1300 (2009).

14. D. J. Durian, "The diffusion coefficient depends on absorption," Opt. Lett. 23, 1502-1504 (1998).

15. I. Freund, "Surface reflections and boundary conditions for diffusive photon transport," Phys. Rev. A 45, 8854-8858 (1992).

16. R. Aronson, "Boundary conditions for diffuse light," J. Opt. Soc. Am. A 12, 2532-2539 (1995).

17. J. Ripoll, M. Nieto-Vesperinas, A. S. R., and H. Deghani, "Boundary conditions for light propagation in diffusive media with nonscattering regions," J. Opt. Soc. Am. A 17, 1671-1681 (2000).

18. C. Chen, J. Du, and L. Pan, "Extending the diffusion approximation to the boundary using an integrated diffusion model," AIP Advances 5, 067115 (2015).

19. S. Fantini, M. A. Franceschini, and E. Gratton, "Effective source term in the diffusion equation for photon transport in turbid media," Appl. Opt. 36, 156-163 (1997).

20. A. F. Kostko and V. A. Pavlov, "Location of the diffusing-photon source in a strongly scattering medium," Appl. Opt. 36, 7577-7582 (1997).

21. X. Intes, B. Le Jeune, F. Pellen, Y. Guern, J. Cariou, and J. Lotrian, "Localization of the virtual point source used in the diffusion approximation to model a collimated beam source," Waves in Random and Complex Media 9, 489-499 (1999).

22. E. M. Sevick-Muraca and C. L. Burch, "Origin of phosphorescence signals reemitted from tissues," Opt. Lett. 19, 1928-1930 (1994).

23. A. Ishimaru, Wave Propagation and Scattering in Random Media (IEEE Press, Piscataway, NJ, 1997).

24. J. R. Lorenzo, Principles of diffuse light propagation (World Scientific, 2012).

25. V. A. Markel and J. C. Schotland, "Symmetries, inversion formulas, and image reconstruction for optical tomography," Phys. Rev. E 70, 056616 (2004).

26. K. M. Case and P. F. Zweifel, Linear Transport Theory (Addison-Wesley, Reading, MA, 1967).

27. S. Chandrasekhar, Radiative Transfer (Dover Pub., New York, 1960).

28. D. J. Durian and J. Rudnick, "Photon migration at short times and distances and in cases of strong absorption," J. Opt. Soc. Am. A 14, 235-245 (1997). 